\title{
Chiral extrapolation of pion-pion scattering phase shifts within standard and unitarized Chiral Perturbation Theory
}

\author{
J. Nebreda, J. R. Peláez, and G. Ríos \\ Departamento de Física Teórica II. Universidad Complutense, 28040, Madrid. Spain \\ (Received 27 December 2010; revised manuscript received 29 March 2011; published 11 May 2011) \\ We calculate the pion-pion elastic scattering phase shifts for pion masses from the chiral limit to values \\ of interest for lattice studies. At low energies, we use the standard Chiral Perturbation Theory expressions \\ to one and two loops. In addition, we study the phase shifts' mass dependence in the resonance region by \\ means of dispersion theory in the form of unitarized Chiral Perturbation Theory and the inverse amplitude \\ method. We pay particular attention to the case when resonances are close to threshold, illustrating the \\ different behavior between scalar and vector resonances. We also provide the estimation of uncertainties, \\ which are dominated by those of the $O\left(p^{6}\right)$ chiral parameters.
}

DOI: 10.1103/PhysRevD.83.094011

PACS numbers: 14.40.Be, 12.39.Fe, 13.75.Lb

\section{INTRODUCTION}

Elastic pion-pion scattering has been an object of study for many decades due to several reasons. In particular, pions are very relevant in the description of final states in other hadronic processes. Also, the two-pion correlated exchange in the scalar-isoscalar channel is the main contribution to nucleon-nucleon attraction, and has been interpreted for long as a scalar "sigma" resonance [1] whose existence, mass, and width have been the subject of an intense debate. Actually, this resonance, nowadays called $f_{0}(600)$, appears as a pole deep in the second Riemann sheet of the scattering amplitude (see the "Note on scalar mesons" in [2] for a detailed account). Finally, the pionpion interaction at low energies is also relevant for the determination of light quark mass ratios and the size of the chiral condensate [3].

On the theory side, unfortunately, neither the elastic resonance region nor the low-energy region are accessible to perturbative QCD calculations. In order to describe these processes in terms of quarks and gluons, one should rely on lattice techniques. For a long time, these techniques have found little applications in this low-energy realm due to complications on the implementation of chiral symmetry, the small physical values of the light quarks and other technicalities as the existence of quarkline disconnected diagrams in some channels. However, very recently, lattice results have become available for the $\rho(770)$ and $f_{0}(600)$ resonance masses [4-8], the pion decay constant, or even the isospin 2 scattering length $[9,10]$, obtained with pion masses which are not too far from the physical values. Recent developments [11] in algorithms may make disconnected diagrams for multihadron calculations tractable in the not too distant future. This means that pion-pion scattering phase-shifts might be calculable soon within lattice-QCD. Actually, some first results for the isospin 2 waves have been obtained for still somewhat large pion masses [12,13]. Of course, lattice calculations still have systematic uncertainties which are hard to estimate and they always rely on modified actions, finite volumes, and other complications so that their physical results are actually extrapolations to the physical limit. It is, therefore, necessary to understand how these chiral or physical extrapolations should be carried out.

Fortunately, even though we cannot rely on perturbative QCD at low energies, we can still use its effective lowenergy theory, known as Chiral Perturbation Theory (ChPT) [14], which provides a rigorous, systematic and model independent expansion of hadronic observables in terms of the external meson momenta and the relatively small pion mass. We will very briefly review ChPT in Sec. II, mostly to introduce the required notation.

Within ChPT, the quark mass dependence appears in a model independent way through the pion mass squared, which is also described as an expansion. Remarkably, the isospin $I=2$ scattering length $m_{\pi}$ dependence found on the lattice is rather well described by just leading order ChPT up to surprisingly large pion masses $[9,10]$, and the one-loop corrections seem to be rather small. In this work we will first study the evolution of the lowest five pion-pion scattering phase-shifts, with definite isospin and angular momentum $(I, J)=(0,0)),(1,1),(2,0),(0,2)$, and $(2,2)$, using the one and two-loop standard ChPT expressions, estimating the uncertainties due to the relatively poor knowledge of the low-energy constants-particularly those at two loops. Of course, this approach is limited to low masses and momenta and cannot be used to describe resonances, although, in principle it should be able to describe their low-energy tails, through, for instance, the low-energy scattering phase-shifts. This is the reason why one of the aims of this work is to study the evolution of all $\pi \pi$ scattering phase-shifts at low-energy within standard ChPT.

Beyond the low-energy regime, it is still possible to obtain the quark mass dependence of hadronic observables, by combining ChPT with dispersion relations. Thus, in Sec. V, we briefly review the inverse amplitude method (IAM) [15-17], obtained by using the elastic 
approximation together with $\mathrm{ChPT}$, to calculate the subtraction constants and the left cut contribution of a dispersion relation for the inverse of the partial waves. This technique provides a description of meson-meson scattering which is simultaneously compatible with the ChPT lowenergy description but also generates the lightest elastic resonance on each channel. By applying this technique to the $\pi \pi$ scattering amplitude to one-loop in SU(2) ChPT, some of us have calculated the pion mass dependence of the $\rho(770)$ and $f_{0}(600)$ masses and widths [18]. Interestingly, this method had already been applied to study only the $f_{0}(600)$ quark mass dependence and its influence, through the nucleon-nucleon interaction, on the production of carbon and oxygen and its anthropic implications [19]. Recently [20], some of us have also calculated the $\kappa(800)$ and $K^{*}(892)$ mass and width dependence with respect to the non strange-quark mass, as well as the dependence of all these four resonances with respect to the strange-quark mass. And even more recently [21], we have extended to two loops the analysis of the $\rho(770)$ and $f_{0}(600)$ resonances within unitarized elastic $\pi \pi$ scattering.

The IAM results for the $m_{\pi}$ dependence of the $\rho(770)$ agree nicely with the estimations for the two first coefficients of its chiral expansion [22], and also with the existing lattice results [4-8]. The comparison with lattice is relatively straightforward in this case since the $\rho(770)$ is not extremely wide and it is actually calculated as a state of the spectrum.

Unfortunately, the comparison of the IAM with lattice results will not be so straightforward for the scalar channels. First, we find of particular interest the repulsive $I=2$ channels. Note that these channels have no resonances, so that neither the spectroscopic studies on the lattice nor our pole studies with the IAM $[18,20,21]$ address this case. However, this is the simplest channel for scattering lattice studies and, as commented above, there are already some lattice results for the scattering length down to relatively low pion masses $[9,10]$ and for phase-shifts but only for $m_{\pi} \simeq 400 \mathrm{MeV}$ or higher [12,13].

Second, we are also interested in the much debated isoscalar channel. Of course, given the status of the $\sigma$ or $f_{0}(600)$, reliable lattice results would be most welcome. Unfortunately, lattice calculations in this channel are hard due to disconnected diagrams, but also their interpretation would be complicated because this resonance is extremely wide (see [2] and references therein). In addition, it was shown in $[18,20]$ that, for sufficiently high masses, the $f_{0}(600)$, being a scalar, becomes a virtual state-a pole in the second Riemann sheet below threshold-which is not a physical state of the spectrum. Therefore, since spectroscopic (or "pole") lattice studies of the $\sigma$ may be rather complicated, a study of the scalar phase shift, as the one presented here, deserves more interest.

These are the motivations to study the chiral extrapolation of phase shifts either from standard or unitarized
ChPT. This will be done first for standard ChPT to next to leading order (NLO) in Sec. III A, and then to next to next to leading order (NNLO) in Sec. III B. Surprisingly, in both cases, the predicted behavior for the phase shift in the $\rho(770)$ may look counterintuitive when compared with present lattice calculations of the $\rho(770)$ mass $m_{\pi}$ dependence. This discussion deserves a separate section, in which we also evaluate the pion mass dependence of the "size" of the $\rho(770)$. Next, we will present the IAM results for NLO ChPT in Sec. VI A and for NNLO in Sec. VIB. We will discuss and summarize all our findings in Sec. VIII.

\section{CHIRAL PERTURBATION THEORY}

Pions are the Goldstone bosons associated to the spontaneous chiral symmetry breaking of QCD. If quarks were strictly massless, pions would be massless too and separated by a gap of the order of $1 \mathrm{GeV}$ from the rest of hadrons, becoming the relevant QCD low-energy degrees of freedom. Chiral Perturbation Theory (ChPT) [14] is nothing but the most general Lagrangian built out as an expansion in pion momenta (i.e., derivatives) respecting the QCD symmetries. In real life, though, the $u$ and $d$ quarks have a very small mass, that we will take in the isospin limit as $\hat{m}=$ $\left(m_{u}+m_{d}\right) / 2$, which can be treated as a perturbation within ChPT. As a consequence, pions have a physical mass of $m_{\pi}=139.57 \mathrm{MeV}$, whose model independent perturbative expansion in terms of $\hat{m}$ is given by ChPT. In summary, the QCD low-energy theory we will use is SU(2) ChPT [14], which corresponds to considering the $u$ and $d$ quarks only and integrating out the other four quarks, whose effect will be included in the low-energy constants (LECs) that multiply each term of the ChPT Lagrangian. In this way, only pions will circulate in the loops. Hence, by varying the pion mass while keeping the ChPT low-energy constants fixed, we are sensitive to the light quark mass dependence for constant $s, c, b$ and $t$ masses.

\section{Perturbative $\pi \pi$ scattering within ChPT}

Pion-pion elastic scattering is customarily described in terms of partial wave amplitudes $t_{J}^{(I)}(s)$ of definite isospin $I$ and angular momentum $J$, where $s$ is the Mandelstam variable, although for simplicity we will drop these indices when there is no possible confusion. From ChPT, these partial waves are obtained as a series expansion $t=t_{2}+t_{4}+t_{6} \cdots$, with $t_{k}=O\left(p / 4 \pi f_{\pi}\right)^{k}$, where $p$ stands generically for center of mass momenta or pion masses. The leading order (LO) $t_{2}$ is $O\left(p^{2}\right)$ and is universal [23] in the sense that it only depends on the scale $f_{\pi} \simeq 92.4 \mathrm{MeV}$ and $m_{\pi}$. The NLO calculation yields $t_{4}$ [14] and is obtained from one-loop diagrams with LO vertices and tree diagrams from the NLO Lagrangian terms, which are multiplied by some low-energy constants (LECs), called $l_{i}^{r}(\mu)$. These LECs absorb the dependence 
TABLE I. ChPT low-energy constants from [24] that contribute to $\pi \pi$ scattering to $O\left(p^{4}\right)$ and $O\left(p^{6}\right)$ that we use in our standard ChPT calculations. The value for $l_{3}^{r}(\mu)$ comes from a recent analysis of the lattice results [25]. The renormalization scale is set to $\mu=770 \mathrm{MeV}$. Errors are only statistical or "only account for the noise seen in the calculations" of [24]. The first four $r_{i}$ and their uncertainties are obtained from resonance saturation. The $r_{f}^{r}(\mu)$ value is from [26].

\begin{tabular}{lccc}
\hline \hline$O\left(p^{4}\right)$ & LECs $\left(\times 10^{-3}\right)$ & $O\left(p^{6}\right)$ & LECs $\left(\times 10^{-4}\right)$ \\
\hline$l_{1}^{r}(\mu)$ & $-3.98 \pm 0.62$ & $r_{1}^{r}(\mu)$ & $-0.60 \pm 0.35$ \\
$l_{2}^{r}(\mu)$ & $1.89 \pm 0.23$ & $r_{2}^{r}(\mu)$ & $1.28 \pm 0.74$ \\
$l_{3}^{r}(\mu)$ & $0.18 \pm 1.11$ & $r_{3}^{r}(\mu)$ & $-1.68 \pm 0.97$ \\
$l_{4}^{r}(\mu)$ & $6.17 \pm 1.39$ & $r_{4}^{r}(\mu)$ & $-1.00 \pm 0.58$ \\
& & $r_{5}^{r}(\mu)$ & $1.52 \pm 0.42$ \\
& & $r_{6}^{r}(\mu)$ & $0.40 \pm 0.04$ \\
& & $r_{f}^{r}(\mu)$ & $0.00 \pm 1.20$ \\
\hline \hline
\end{tabular}

on the loop regularization scale $\mu$, and are determined by the underlying QCD dynamics. Their measured values can be found in Table I. Something similar happens with the NNLO result $t_{6}$ [27], which has two-loop contributions with LO vertices, one-loop contributions with one LO vertex and one NLO vertex containing some $l_{i}$, plus tree level diagrams with NNLO vertices, whose LECs appear only in six combinations now called $r_{i}^{r}(\mu)$, whose estimated values are listed also in Table I. All these LECs carry a scale dependence that cancels that from loop integrals, so that observables are scale independent and finite order by order.

Let us remark that we write the $\pi \pi$ scattering amplitude in terms of the physical constants $m_{\pi}$ and $f_{\pi}$, which are obtained as expansions in powers of the LO pion mass. Actually, $l_{3}$ and $l_{4}$ appear at NLO in $\pi \pi$ scattering through these $m_{\pi}$ and $f_{\pi}$ expansions, but in contributions that depend stronger on the pion mass and softer on the energy than those containing the other LECs. Thus, $l_{3}$ and $l_{4}$ are harder to determine experimentally and have the largest uncertainty. This is particularly severe for $l_{3}$, and that is why we have used its lattice determination [25] quoted in Table I.
At NNLO, the expansion of $f_{\pi}$ on the physical pion mass [28] requires an additional parameter $r_{f}$, also listed in Table I. Note that there is an additional $O\left(p^{6}\right)$ constant, $r_{M}$, which appears in the NNLO chiral expansion of the physical pion mass $m_{\pi}$ in powers of the quark mass $\hat{m}$, but such a constant would only be needed in order to study the quark mass dependence of observables. However, quark masses carry some renormalization scale and scheme dependence and most lattice results provide their results in terms of the physical pion mass. That is why here we will study the dependence of scattering phases on the physical pion mass and not on the quark mass. Therefore, we do not need $r_{M}$.

We show in Table I the estimated statistical uncertainties of the LECs (for $r_{5}, r_{6}$ they are described as the noise in the dispersive calculation of [24]). Systematic uncertainties are large and harder to estimate; for illustration, we also provide in Table II other values found in the literature at $O\left(p^{4}\right)$ and $O\left(p^{6}\right)$. We consider the spread on these values as a crude indication of the size of systematic uncertainties. From the sets in [30] we note that, even for the same analysis, the values of the $O\left(p^{4}\right)$ LECs can be somewhat different whether they are obtained from a pure $O\left(p^{4}\right)$ calculation or including the $O\left(p^{6}\right)$ corrections. Hence, it should not come as a surprise later that the $O\left(p^{6}\right)$ values obtained from a unitarized fit, which includes part of the higher order corrections, may also come out somewhat different from the values obtained in a pure ChPT $O\left(p^{6}\right)$ analysis.

As a final comment concerning ChPT parameters, it is possible and usual to write the NNLO $\pi \pi$ scattering amplitude in terms of just six parameters $b_{1}, \ldots, b_{6}$, multiplying each one of the energy dependent polynomials allowed by Lorentz invariance and chiral symmetry. Thus, the knowledge of 6 constants is enough to describe $\pi \pi$ scattering to that order. However, these $b_{i}$ parameters do carry a dependence on $m_{\pi}$ and the full knowledge of all the $l_{i}$ and $r_{i}$ constants is needed to extrapolate to unphysical values of $m_{\pi}$, which is the object of this work, and the reason why we need to determine 11 parameters instead of just six.

TABLE II. Samples of other sets of LECs: First row: SU(3) analysis of $\pi K$ scattering using Roy-Steiner equations. Second and third rows: $K_{14}$ analysis to $O\left(p^{4}\right)$ and $O\left(p^{6}\right)$, respectively. Naively, we have combined quadratically the SU(3) LECs errors there. Fourth row: Roy equations analysis. Uncertainties from imaginary parts and unknown $O\left(p^{6}\right)$ LECs combined quadratically. Last row, values used in [18] with the one-loop IAM. All LECs are evaluated at the scale $\mu=770 \mathrm{MeV}$.

\begin{tabular}{lcccc}
\hline \hline Analysis & $10^{3} l_{1}^{r}$ & $10^{3} l_{2}^{r}$ & $10^{3} l_{3}^{r}$ & $10^{3} l_{4}^{r}$ \\
\hline ChPT $O\left(p^{4}\right)[29]$ & $-4.9 \pm 0.6$ & $5.2 \pm 0.1$ & & $17 \pm 10$ \\
ChPT $O\left(p^{4}\right)[30]$ & -4.5 & 5.9 & 2.1 & 5.7 \\
ChPT $O\left(p^{6}\right)[30]$ & $-3.3 \pm 2.5$ & $2.8 \pm 1.1$ & $1.2 \pm 1.7$ & $3.5 \pm 0.6$ \\
ChPT $O\left(p^{6}\right)[31]$ & $-4.0 \pm 2.1$ & $1.6 \pm 1.0$ & & \\
IAM $O\left(p^{4}\right)[18]$ & $-3.7 \pm 0.2$ & $5.0 \pm 0.4$ & $0.8 \pm 3.8$ & $6.2 \pm 5.7$ \\
\hline \hline
\end{tabular}


Now, elastic unitarity implies for partial waves, at physical values of $s$, that:

$$
\operatorname{Im} t(s)=\sigma(s)|t(s)|^{2} \Rightarrow \operatorname{Im} 1 / t(s)=-\sigma(s),
$$

where $\sigma(s)=2 p / \sqrt{s}, p$ being the center of mass momentum. As a consequence, the modulus of $t(s)$ is related to its phase:

$$
t(s)=|t(s)| e^{i \delta(s)}=e^{i \delta(s)} \sin \delta(s) / \sigma(s) .
$$

This "phase-shift" $\delta(s)$, which determines completely the amplitude, is the usual way to parametrize partial waves that we will use next to predict the amplitude variation when the pion mass is changed. Of course, before extrapolating to other pion masses, we will compare the ChPT amplitudes, with and without unitarization, with the existing experimental data.

ChPT amplitudes, being an expansion, satisfy unitarity only perturbatively:

$$
\operatorname{Im} t_{2}=0, \quad \operatorname{Im} t_{4}=\sigma\left|t_{2}\right|^{2}, \quad \operatorname{Im} t_{6}=2 t_{2} \operatorname{Re} t_{4} \ldots
$$

In particular, ChPT partial waves are expected to violate unitarity as $s$ increases, since they are basically polynomials in $s$. In Sec. V, we will use ChPT inside dispersion relations to obtain amplitudes that, while respecting the ChPT expansion at low energies, satisfy unitarity and allow and provide a good description of experiment up to higher energies.

After this brief introduction to ChPT and its notation, we are now ready to present our first calculations.

\section{RESULTS WITHIN STANDARD CHPT}

Using the equations above, the phase shift within standard ChPT is obtained as a series expansion (see [32] for a prescription on how to perform this expansion):

$$
\begin{aligned}
& \delta=\sigma\left(t_{2}+\operatorname{Re} t_{4}\right)+O\left(p^{6}\right), \\
& \delta=\sigma\left(t_{2}+\operatorname{Re} t_{4}+\operatorname{Re} t_{6}\right)+\frac{2}{3}\left(\sigma t_{2}\right)^{3}+O\left(p^{8}\right),
\end{aligned}
$$

which are the expressions used in our one-loop and twoloop calculations, respectively, that we detail next.

Now, let us recall that the pion-and quark-mass dependence of the partial waves $t(s)$ within ChPT comes from two different sources: from kinematics, through pion propagators, or from the dynamics encoded in the vertices. In particular, the threshold shift is purely of a kinematic nature and rather trivial to understand. Therefore, although $\pi \pi$ phase shifts are customarily presented in terms of $\sqrt{s}$, we are showing them here as a function of the center of mass momentum $p$, which is also more convenient to compare to lattice studies. With this kinematic threshold effect "subtracted", the remaining $m_{\pi}$ dependence is rather mild for most partial waves. As we will see, this soft dependence of the $\delta_{I J}(p)$ on $m_{\pi}$ has been also found for $I=2$ waves in very recent lattice calculations [12].

\section{A. One-loop ChPT}

In Fig. 1 we show the phase shifts from the one-loop ChPT, i.e. $O\left(p^{4}\right)$, for the $(I, J)=(0,0),(2,0),(1,1) \pi \pi$ scattering waves. Note that for the $(1,1)$ channel, the description fails much before $p \simeq 300 \mathrm{MeV}$. This momentum is typically below the $\rho(770)$ resonance region, which is a natural applicability bound for the ChPT series. This resonance has a relatively narrow shape, corresponding to a pole close to the real axis in the second Riemann sheet, which, of course, is completely missed by one-loop ChPT except in its very low-energy tail. In contrast, one-loop ChPT is giving a fairly good description of the $(0,0)$ channel even up to, say $p=350$ or $400 \mathrm{MeV}$. In this case, there is also a resonance-the scalar $\sigma$ (or $f_{0}(600)$ ) - but it is very wide and its pole is deep in the complex plane, so that it is not seen in the real axis as the typical sharp rise in the phase. For this reason, and despite being an expansion which has no such a pole in the complex plane, ChPT results are not very different qualitatively from the data in this channel. Finally, we see that the oneloop description of the $(2,0)$ channel is also reasonably good up to such high momentum, mostly due to the fact that this channel has no resonances and also that the data are not particularly precise.

The gray areas in the figure cover the uncertainties due to the statistical error in the LECs detailed in the previous section. In order to calculate these areas we have used a Monte Carlo sampling. For each phase-shift calculation we have generated 5000 different samples of LECs using a Gaussian distribution with variances equal to the errors quoted in Table I. To avoid a confusing overlapping between uncertainty bands, we only show the one corresponding to the physical pion mass. In the appendix we provide a detailed study of the evolution of these uncertainties with $m_{\pi}$. As a general feature for both scalar and vector waves, the relative uncertainty of the phase at a given momentum grows slowly with $m_{\pi}$.

Once we have checked where one-loop ChPT calculations provide an acceptable description of data, we can now compare, also in Fig. 1, with the results obtained if we change the pion mass from its physical value to $m_{\pi}=230$, 300 and $350 \mathrm{MeV}$. The first observation is that the sign of the phase derivative does not change when increasing the pion mass, at least up to $350 \mathrm{MeV}$, which means that the attractive or repulsive nature of each wave is conserved.

In that figure, we have represented with an arrow the direction of the phase movement as $m_{\pi}$ increases. Thus, the next observation is that both scalar phase shifts increase in absolute value as $m_{\pi}$ grows, whereas the phase of the vector channel decreases.

The behavior of the phase at low momentum in the vector channel may seem surprising at first, because 

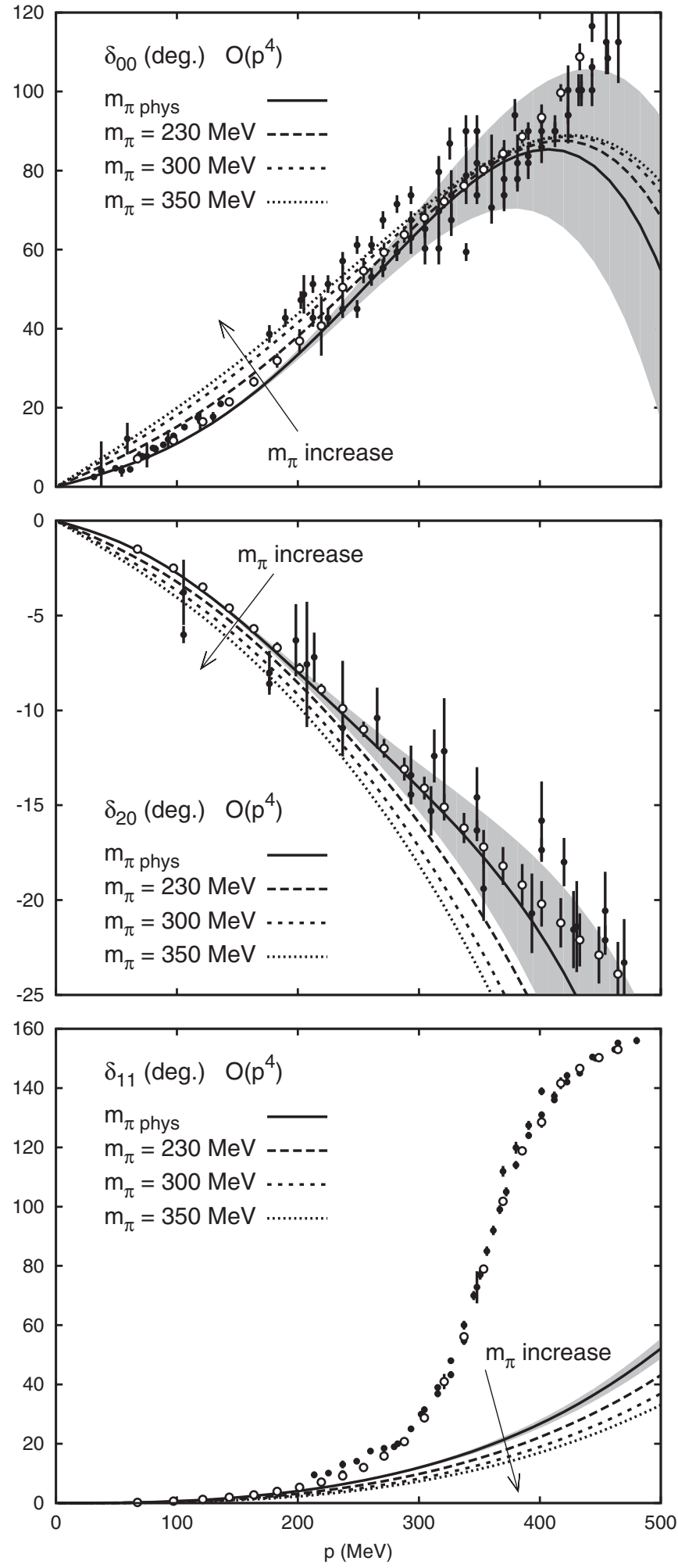

FIG. 1. $\quad S$ and $P$ wave $\pi \pi$ phase shifts from standard ChPT up to one loop. Different lines stand for different pion masses $m_{\pi}=139.57,230,300$ and $350 \mathrm{MeV}$, respectively. Since the lines are too close to each other, we only show error bands for the physical mass. Experimental data come from [33] (black circles) and the precise model independent dispersive data analysis from [34] (white circles). The arrows show the direction of increasing $m_{\pi}$. several lattice works [4-8], the chiral effective treatment [22], as well as the IAM [18], predict that the $\rho(770)$ mass increases much slower than the $2 \pi$ threshold as $m_{\pi}$ grows. But then, when the $\rho(770)$ peak reaches a given momentum, the phase there should be $\pi / 2$ to a very good approximation. Therefore, one would expect naively the phase at low momentum to rise as $m_{\pi}$ grows. However, the model independent ChPT analysis tells us otherwise. We will see in detail in Sec. IV why this intuitive picture fails and the phase shift actually has to decrease at first and increase later on.

Finally, in Fig. 2, we show the one-loop ChPT results for the $D$ waves: $(I, J)=(0,2)$ and $(2,2)$. We show these separately because both of them vanish at $O\left(p^{2}\right)$, so that the one-loop $O\left(p^{4}\right)$ calculation is just their LO contribution. Actually, they are both very small at low energies.
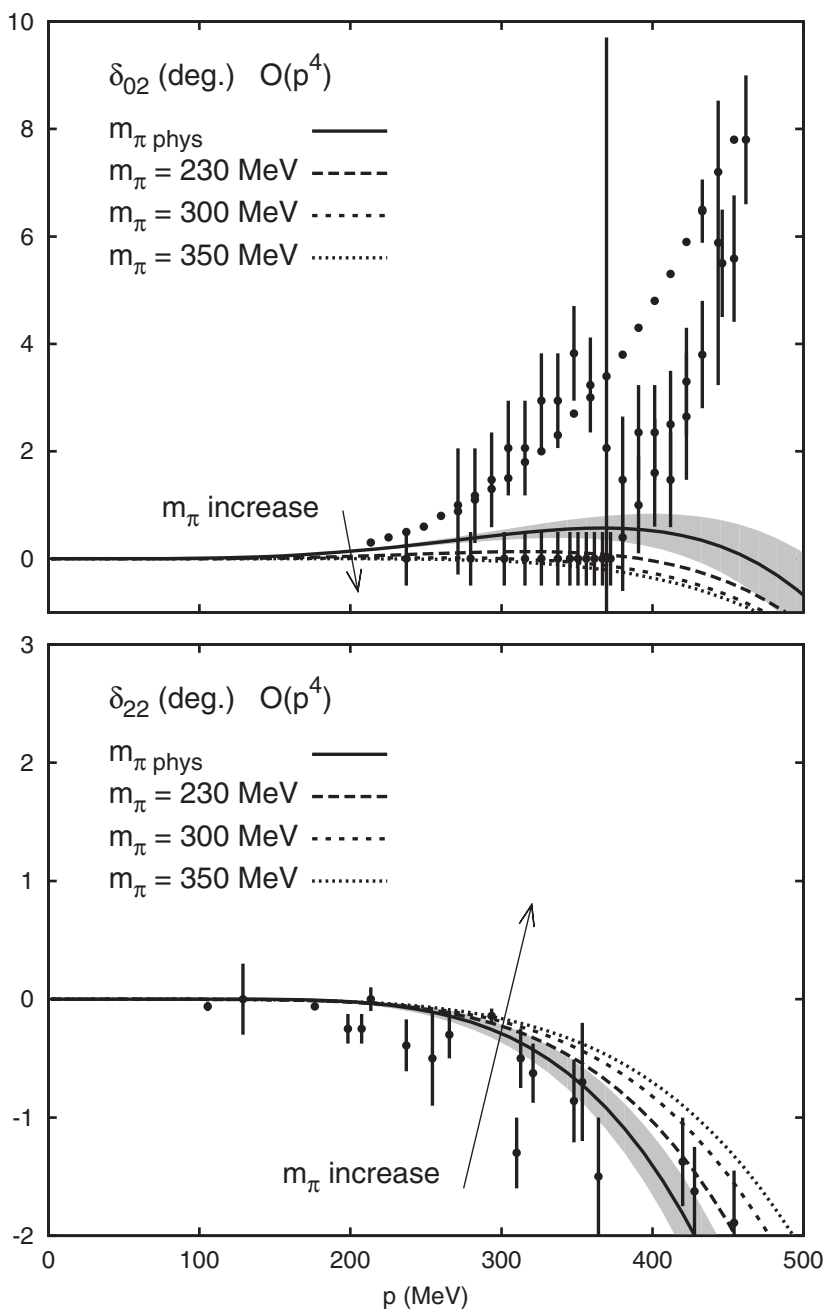

FIG. 2. $D$ wave $\pi \pi$ phase shifts from standard ChPT up to one loop. Different lines stand for different pion masses $m_{\pi}=$ 139.57, 230, 300 and $350 \mathrm{MeV}$, respectively. Since the lines are too close to each other, we only show error bands for the physical mass. Experimental data come from [33]. The arrows show the direction of increasing $m_{\pi}$. 
We can see in the figures that the one-loop ChPT calculation provides an acceptable solution for the $(2,2)$ wave up to relatively high momentum, but obviously it cannot reproduce the resonance shape of the $f_{2}(1270)$ resonance in the $(0,2)$ channel. As before, we only show the uncertainty band due to the statistical errors on the LECs for the physical pion mass, obtained again from a Monte Carlo Gaussian sample. Relative uncertainties for different pion masses are detailed in the appendix.

Note that, in contrast to the scalar waves, both tensor phase shifts decrease in absolute value as the pion mass increases not too far from its physical value. In this sense, they are more similar to the vector channel behavior. Remarkably, for larger pion masses and momentum the $(0,2)$ phase shift even changes sign and the derivative becomes negative. However, this behavior is not found at two loops, as we will see in the next subsection.

\section{B. Two-loop ChPT}

We use the two-loop $\pi \pi$ scattering calculation in [27]. Note, however, that instead of the usual $b_{1} \ldots b_{6}$ parameters, in order to implement the $m_{\pi}$ dependence we need to use the one-loop $l_{1} \ldots l_{4}$ and $r_{i}$ parameters in Table I. In Fig. 3 we show the resulting phase shifts for the $(I, J)=$ $(0,0),(1,1)$, and $(2,0)$ waves for the physical $m_{\pi}$ but also for $m_{\pi}=230,300$, and $350 \mathrm{MeV}$.

The uncertainty bands, which we show only for the physical pion mass - see the appendix for other massesare once again calculated with a Monte Carlo Gaussian sampling of 5000 sets of LECs, using as standard deviations the uncertainties quoted in Table I. The only exception are the $r_{1 \ldots 4}$ parameters, which are estimated from resonance saturation and, as in [24], we have assumed that all values in the interval from 0 to twice the estimation are equally likely. Of course, we want to emphasize that this is just an estimate of the values of the $O\left(p^{6}\right)$ parameters, which are rather difficult to determine. Possible improvements in their determinations could come from future lattice-QCD calculations, as it has already been done with the $O\left(p^{4}\right)$ LECs (see [25] for a review) or from the use of recent dispesive data analysis like that in [34] inside threshold parameter sum rules [35].

Also, since the renormalization scale $\mu$ where the estimates for $r_{1 \ldots 4}$ and $r_{f}$ apply is not known, another source of uncertainty appears. Our calculations are made at $\mu=$ $770 \mathrm{MeV}$ so, in order to account for the uncertainty due to that choice, we have followed [24] again and we have calculated the shift occurring in the phase shift if $r_{1 \ldots 4}$ are fixed and the scale is changed to $\mu=500 \mathrm{MeV}$ and $\mu=$ $1 \mathrm{GeV}$. That shift is added in quadrature to the errors given by the Monte Carlo sampling.

The general features of the one-loop description still apply to the two-loop case. Namely, all waves keep their attractive or repulsive nature, and both scalar phases increase in absolute value as $m_{\pi}$ grows, whereas the vector
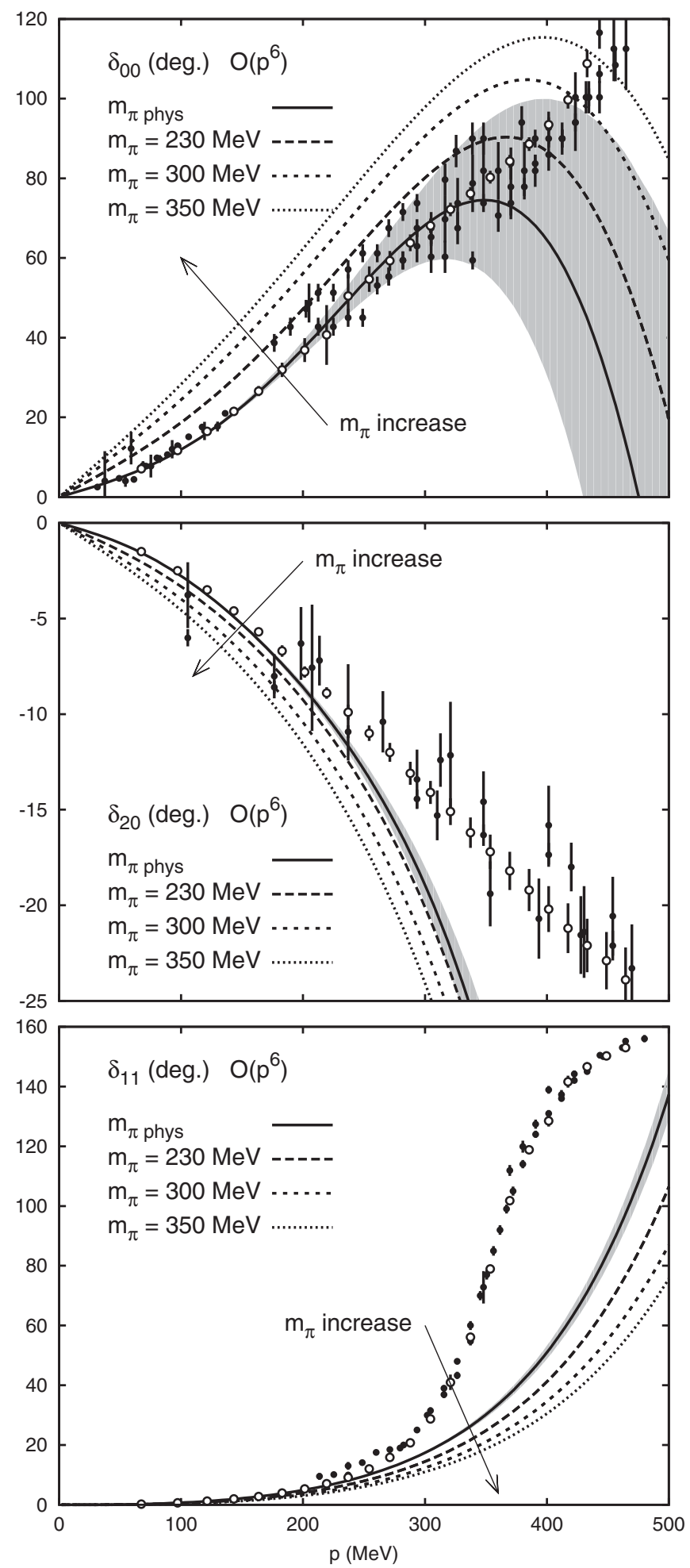

FIG. 3. $S$ and $P$ wave $\pi \pi$ phase shifts from standard ChPT up to two loops. Different lines stand for different pion masses: continuous, long dashed, short dashed and dotted for $M_{\pi}=$ 139.57, 230, 300 and $350 \mathrm{MeV}$, respectively. Since the lines are too close to each other, we only show error bands for the physical mass. Experimental data (black circles) come from [33] and the precise model independent dispersive data analysis from [34] (white circles). The arrows show the direction of increasing $m_{\pi}$. 
channel phase decreases. The counterintuitive behavior of the $\rho(770)$ is therefore a robust prediction of ChPT. In the next section, we will explain with a simple model why chiral symmetry requires this behavior. Still, the description of the $(0,0)$ wave is fair only up to $p=300$ or $350 \mathrm{MeV}$, although it has improved remarkably in the low-energy region, where the data are most recent and reliable, as they come from $K_{\ell 4}$ decays. The $(1,1)$ phase is now much closer to the experimental data, and thus it seems to provide a fairly good representation up to, say $p=200 \mathrm{MeV}$. However, the description of the $(2,0)$ has deteriorated for higher momenta, and seems to be good only up to, roughly, 200 or $250 \mathrm{MeV}$.

However, despite the qualitative $m_{\pi}$ dependence being similar to the one-loop case, quantitatively the effect is
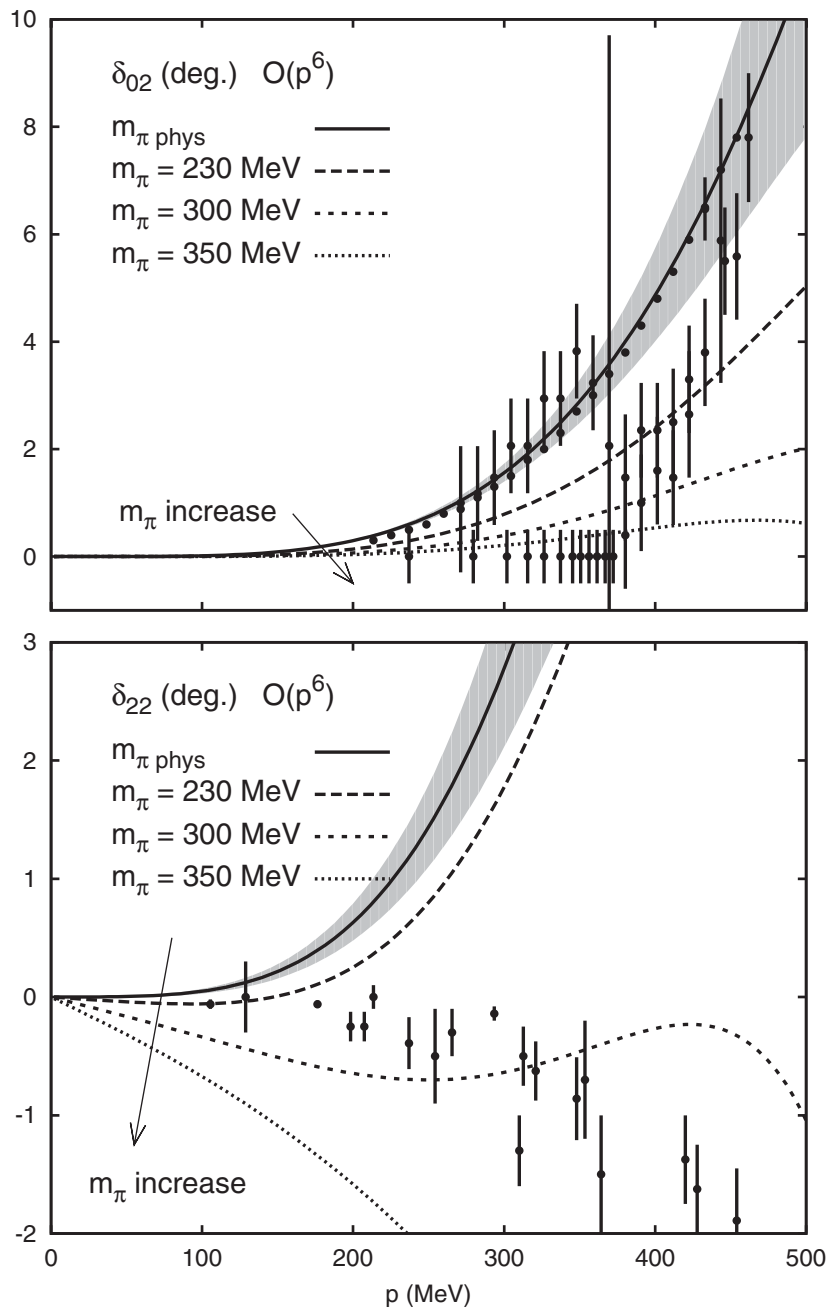

FIG. 4. $D$ wave $\pi \pi$ phase shifts from standard ChPT up to two loops. Different lines stand for different pion masses: continuous, long dashed, short dashed and dotted for $m_{\pi}=139.57,230$, 300 and $350 \mathrm{MeV}$, respectively. Since the lines are too close to each other, we only show error bands for the physical mass. Experimental data (black circles) come from [33]. The arrows show the direction of increasing $m_{\pi}$. stronger. In absolute value all S-wave phase shifts grow faster with $m_{\pi}$ to two loops than they did to one loop.

In Fig. 4, we show the two-loop result for the $\mathrm{D}$ waves. As commented before, these waves have no $O\left(p^{2}\right)$ term, so, this $O\left(p^{6}\right)$ calculation is just a next to leading order calculation. We can see that the differences with the one-loop case are dramatic. The $(I, J)=(0,2)$ phase suffers a remarkable improvement, being able to describe the tail of the $f_{2}(1275)$ resonance up to momentum of the order of $450 \mathrm{MeV}$. Contrary to the one-loop case, within the $m_{\pi}$ range of this study, the $(0,2)$ phase does not become negative. Finally, the $(2,2)$ phase shift fails to describe even the sign of the data, and is only relatively close to the data points below $150 \mathrm{MeV}$. Furthermore, the one-loop $m_{\pi}$ phase-shift dependence was opposite to the two-loop case: from more negative to less negative for the former versus from positive to negative for the second. The predictions for this channel are therefore not very robust, which is also corroborated by the large uncertainties for higher $m_{\pi}$ that can be found in the appendix.

\section{Comparison with lattice results for $I=2$ and $m_{\pi}>350 \mathrm{MeV}$}

As we have already commented, there are very recent lattice results on phase shifts for the $I=2, J=0[12,13]$ and $J=2$ channels [12]. In Figs. 5 and 6 we compare the one and two-loop calculations within standard ChPT, first for the physical mass versus experimental data, and then for $m_{\pi}=396,420,444$, and $524 \mathrm{MeV}$, versus lattice results.

When we examine Fig. 5, corresponding to the $I=2$, $J=0$ phase shifts, the first observation is that all lattice points with $p<200 \mathrm{MeV}$ are well described within the uncertainties of one-loop ChPT, even up to $m_{\pi}=$ $444 \mathrm{MeV}$. From the figure, we observe that a pion mass of $524 \mathrm{MeV}$ seems out of reach and will not be considered any longer. Beyond that momentum, the ChPT calculation bends downwards and misses all other lattice results with higher momenta. Remarkably, the two-loop ChPT results do not improve this agreement. Actually, the two-loop calculation describes somewhat worse the lattice data and seems to move consistently to more negative values than those observed on the lattice, as $m_{\pi}$ grows higher. Let us remark that the curvature downwards is larger in the twoloop result than just to one loop. In view of the figures it seems that the standard ChPT applicability limit is, at best, somewhere around $p \simeq 150-200 \mathrm{MeV}$, up to $m_{\pi}$ of the order of 400-440 MeV.

Unfortunately, for the $I=2, J=2$ channel, shown in Fig. 6, there are no lattice results available at low momentum. Surprisingly, the one-loop calculation agrees quite nicely with the lattice values up to around $p \simeq 500 \mathrm{MeV}$, even for the highest pion mass. However, the two-loop results show a very strong $m_{\pi}$ dependence that is in complete disagreement with the behavior predicted by the 

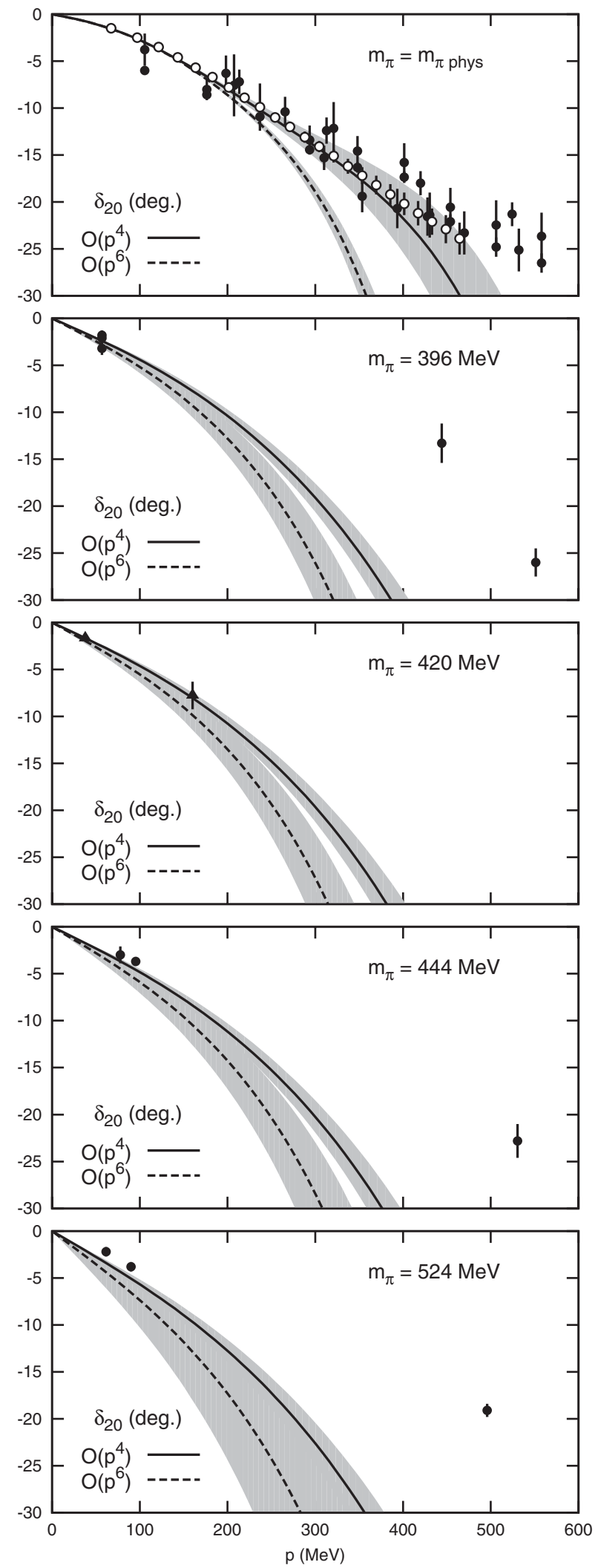

FIG. 5. One and two-loop standard ChPT phase shifts for the $I=2, J=0$ channel. Top panel: results compared to data from [33] (black circles) and [34] (white circles). Rest of panels: results compared to lattice results coming from [12] (circles) and [13] (triangles).
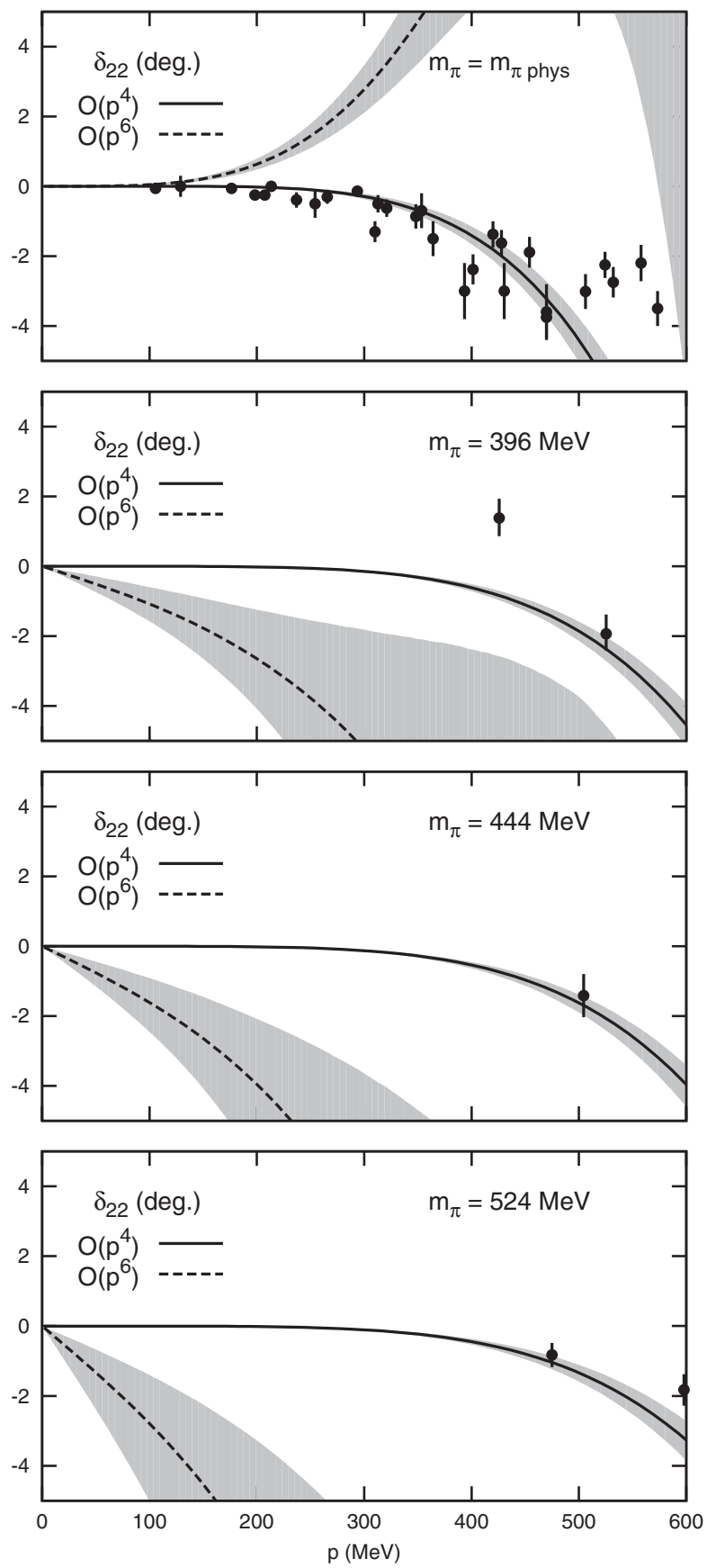

FIG. 6. One and two-loop standard ChPT phase shifts for the $I=2, J=2$ channel. Top panel: results compared to data from [33] (black circles). Rest of panels: results compared to lattice results coming from [12] (circles). Note the large difference between one and two-loop results.

lattice simulations. Even the tendency is wrong, since the absolute value of the phase seems to grow with $m_{\pi}$, whereas lattice results may suggest a decrease. Let us, nevertheless, recall that for $D$-waves the tree level amplitude vanishes, so that one and two-loop calculations correspond only to leading and next to leading order results. Higher order calculations may be needed to improve and stabilize the $D$ wave description. 


\section{RECONCILING THE PHASE SHIFT AND RESONANCE BEHAVIOR IN THE VECTOR CHANNEL}

We have seen that, within ChPT, the low momentum phase shift of the vector channel is found to decrease as $m_{\pi}$ grows. This is a model independent result and looks rather robust since it is obtained both at one and two loops. However, lattice results [4-8], the chiral effective treatment [22], as well as the IAM [18] predict that, in terms of momentum, the $\rho(770)$ peak gets closer and closer to threshold. Thus, for any low momentum choice, and as $m_{\pi}$ increases, the $\rho(770)$ peak reaches that given momentum so that the phase there should be $\pi / 2$. Therefore, one would naively expect the phase shift for any fixed low momentum to grow with $m_{\pi}$.

Actually, this is what one would find if, to describe the $\rho(770)$ resonance pole, one uses the very simple and intuitive (but, as we will see below, incomplete) BreitWigner model

$$
t(s)=\frac{-\sqrt{s} M \Gamma(p) / 2 p}{s-M^{2}+i M \Gamma(p)}
$$

where, $p^{2}=s / 4-m_{\pi}^{2}$ and the width is:

$$
\Gamma(p)=\Gamma_{R}\left(\frac{p}{p_{R}}\right)^{3},
$$

where $M$ is the resonance mass and $p_{R}$ is the pion momentum at the resonance energy $p_{R}^{2}=M^{2} / 4-m_{\pi}^{2}$ so that $t(s)$ behaves correctly at threshold, $t(s) \sim p^{2 l}$. Note that $\Gamma_{R}=g^{2} p_{R}^{3} / 6 \pi M^{2}$ is the $\rho(770)$ decay width.

For the sake of simplicity, let us now assume that the resonance mass $M$ and coupling remain constant when changing the pion mass $m_{\pi}$. This implies that $M$ and $\Gamma(p)$ are $m_{\pi}$ independent. For our illustration purposes here, this is a fairly good approximation to what has been found on the lattice or with the IAM, and it could be considered as the leading order term in the $m_{\pi}$ expansion (see [36] for the $\rho(770)$ mass).

In such case, however, the phase-shift $m_{\pi}$ dependence near threshold does not follow what is obtained from ChPT (or the IAM, as we will see below). In particular, since

$$
\tan \delta(p)=-\frac{M \Gamma(p)}{4 p^{2}-4 p_{R}^{2}},
$$

the only $m_{\pi}$ dependence in $\delta$ (for a given $p$ ) is through $p_{R}$ (and $\left.d\left(p_{R}^{2}\right) / d\left(m_{\pi}^{2}\right)=-1\right)$ so that

$$
\frac{\partial \delta}{\partial\left(m_{\pi}^{2}\right)}=-\frac{\partial \delta}{\partial\left(p_{R}^{2}\right)}=\frac{4 M \Gamma(p)}{\left(4 p^{2}-4 p_{R}^{2}\right)^{2}+M^{2} \Gamma(p)^{2}}>0 .
$$

However, in ChPT, for low $p$ we have shown in Figs. 1 and 3 that $\partial \delta / \partial\left(m_{\pi}^{2}\right)<0$.

Of course, it is very well known that a simple BreitWigner vector formalism is not consistent with the chiral expansion unless there are some additional low-energy contributions - or contact terms in the Lagrangian formalism [37]. Just to keep things very simple we can use a modification of the Breit-Wigner parametrization, which is widely used in analysis of $\pi \pi$ scattering and other phenomenology involving decays into light mesons [38], and reads

$$
\Gamma(p)=\Gamma_{R}\left(\frac{p}{p_{R}}\right)^{2 l+1} \frac{D_{l}\left(p_{R} r\right)}{D_{l}(p r)} \equiv \tilde{\Gamma}(p) \frac{D_{l}\left(p_{R} r\right)}{D_{l}(p r)} .
$$

Here $\tilde{\Gamma}(p)$ is $m_{\pi}$ independent and $D_{l}(p r)$ are the BlattWeisskopf centrifugal barrier functions [39], that for $l=1$ read $D_{1}(p r)=1+(p r)^{2}$. All the $m_{\pi}$ dependence is carried by $p_{R}$ and the new parameter $r$, which is usually interpreted as a crude estimate of the size of the meson, although it should not be identified with its mean square charge radius. At low momentum we now find

$$
\frac{\partial \delta(p)}{\partial\left(m_{\pi}^{2}\right)} \simeq \frac{1+p_{R}^{4}\left(r^{2}\right)^{\prime}}{4 p_{R}^{4}} M \tilde{\Gamma}(p),
$$

where $\left(r^{2}\right)^{\prime}$ stands for $d r^{2} / d\left(m_{\pi}^{2}\right)$. In order to have a decreasing phase shift at low $p$ when increasing $m_{\pi}$, we just need $1+p_{R}^{4}\left(r^{2}\right)^{\prime}<0$. We will see below that this is actually required by chiral symmetry at leading order in the pion mass expansion. This would explain the phase decrease seen in ChPT for not too large $m_{\pi}$, even though the $\rho(770)$ is approaching threshold as $m_{\pi}$ grows. Of course, when $m_{\pi}$ grows too large, and particularly in the limit when the $\rho(770)$ tends to threshold, so that $p_{R} \rightarrow 0$, the derivative is positive, and the phase shift increases, as one would have expected naively.

Let us then check that chiral symmetry actually requires $1+p_{R}^{4}\left(r^{2}\right)^{\prime}<0$, at least for low pion masses. We can estimate the leading $m_{\pi}$ dependence of $r$ by comparing the low momentum and mass expansion of the amplitude in Eqs. (5) using (9), with that of ChPT. In particular, since in this simple model we have only one parameter, $r$, we will only compare the scattering lengths. Our aim is just to reproduce the leading order $m_{\pi}$ dependence, since we have already made additional approximations and simplifications (like the constancy of the $\rho(770)$ mass and coupling). We define the scattering length, $a$, as $\operatorname{Re} t \simeq p^{2}\left(a+b p^{2}+\cdots\right)$. The low $p$ expansion of the amplitude in Eq. (5) using (9) leads to

$$
\begin{aligned}
a_{B W} & =\frac{m_{\pi} M \Gamma_{R}\left(1+\left(p_{R} r\right)^{2}\right)}{4 p_{R}^{5}} \\
& =\frac{m_{\pi} \Gamma_{R}}{M p_{R}^{3}}\left(1+\frac{1}{4} M^{2} r^{2}+O\left(m_{\pi}^{2}\right)\right) .
\end{aligned}
$$

This result has to be compared with that of ChPT: $a_{\mathrm{ChPT}}=$ $1 / 24 \pi f_{\pi}^{2}+O\left(m_{\pi}^{2}\right)$. Matching with ChPT we obtain for $r^{2}$

$$
r^{2}=\frac{p_{R}^{3}}{6 \pi f_{\pi}^{2} M \Gamma_{R}} \frac{1}{m_{\pi}}+O\left(m_{\pi}^{0}\right)
$$




$$
\equiv \frac{1}{g^{2} f_{\pi}^{2}} \frac{M}{m_{\pi}}+O\left(m_{\pi}^{0}\right) \simeq\left(4.3 \mathrm{GeV}^{-1}\right)^{2} .
$$

The value obtained with this ChPT estimation is compatible with what is found in the literature $\left(r \sim 4-5 \mathrm{GeV}^{-1}\right)$ [38].

Note that the size $r$ explodes as $m_{\pi} \rightarrow 0$. However, this is a very well known feature of hadrons, at least for the charge radius. Actually, the squared charged radius of the pion and the nucleon show a $\log m_{\pi}^{2}$ singularity [14,40,41] and the Pauli radius of the nucleon an additional $1 / m_{\pi}$ singularity [40]. Nevertheless, as we have commented, our $r^{2}$ parameter should not be directly identified with the $\rho(770)$ charged radius, although our results suggest that they may have a similar singularity.

With this $m_{\pi}$ dependence for $r$ we find that

$$
1+p_{R}^{4}\left(r^{2}\right)^{\prime}=1-\frac{M p_{R}^{4}}{2 g^{2} f_{\pi}^{2} m_{\pi}^{3}},
$$

which is negative for the physical values of the parameters. This guarantees that $\partial \delta(p) / \partial\left(m_{\pi}^{2}\right)<0$ for $m_{\pi}$ not far from $m_{\pi}^{\text {phys }}$, and sufficiently low $p$, as is obtained in ChPT.

The decrease is a robust feature of ChPT, although the pure chiral expansion cannot reproduce the $\rho(770)$ resonance. Of course, the model we have presented here is very simple and naive, but provides a qualitative and intuitive explanation of why chiral symmetry implies that the vector phase-shift at low momenta first decreases, although it may increase later as $m_{\pi}$ grows. This model cannot be pushed too far. In particular, we cannot reproduce the chiral behavior of the scattering length beyond leading order or even the slope parameter.

It is, however, possible to incorporate simultaneously the $\rho(770)$ pole and the full low-energy ChPT expansion to one and two loops. In the next section, we will explain the technique in detail and later on we will show how it describes the existing lattice data up to much higher momentum than standard ChPT. Actually, we will check how the vector phase-shift decreases first and then increases as $m_{\pi}$ grows.

\section{UNITARIZED CHPT: THE INVERSE AMPLITUDE METHOD}

As we have already commented in Sect. II, the partial waves obtained from the ChPT expansion are basically a truncated series in momenta or energies and cannot satisfy elastic unitarity, Eq. (1), exactly, but only perturbatively, as in Eq. (3).

There is, however, a well known technique, known as unitarization, to obtain expressions for partial waves that satisfy elastic unitarity, have the correct analytic structure in terms of cuts in the complex plane, and simultaneously respect the ChPT expansion up to a given order. Here we will make use of the elastic inverse amplitude method (IAM) — or a slightly modified version — that implements the fully renormalized one or two-loop ChPT expansion at low energies but does not introduce any spurious parameter in the unitarization procedure. Had we used other, possibly simpler but very successful, unitarization techniques with spurious parameters, like cutoff or any other regulator, we should have had to worry about the unknown $m_{\pi}$ dependence of that scale.

The IAM [17] uses elastic unitarity and the ChPT expansion to evaluate a once subtracted dispersion relation for the inverse amplitude. The analytic structure of $1 / t$ consists on a right cut from threshold to $\infty$, a left cut from $-\infty$ to 0 , and possible poles coming from zeros of $t$. We can write then a once subtracted dispersion relation for $1 / t$, the subtraction point being $s_{A}$,

$$
\begin{aligned}
\frac{1}{t(s)}= & \frac{s-s_{A}}{\pi} \int_{R C} d s^{\prime} \frac{\operatorname{Im} 1 / t\left(s^{\prime}\right)}{\left(s^{\prime}-s_{A}\right)\left(s^{\prime}-s\right)}+L C(1 / t) \\
& +P C(1 / t),
\end{aligned}
$$

where $L C(1 / t)$ stands for a similar integral over the left cut and $P C(1 / t)$ is the contribution of the pole at $s_{A}$. The choice of $s_{A}$ is, in principle, arbitrary, but since we want to use the information encoded in the ChPT series, we are then limited to the low-energy region, preferably, below threshold. Now, scalar waves vanish at the so called Adler zero that lies in the real axis below threshold and in practice this is a very convenient choice for $s_{A}$, which has actually motivated our notation. For other waves, there is no such an Adler zero, and the subtraction point can be taken, for instance, at $s=0$. It is important to remark that the choice of subtraction point, as long as it lies between the left and right cut, has only a very small numerical effect [17] on the physical region. Up to here everything is exact. The most relevant observation is that, following Eq. (1), on the elastic cut we know exactly $\operatorname{Im} 1 / t=-\sigma$.

Now we are going to derive the IAM within one-loop $\mathrm{ChPT}$. First, the Adler zero position can be approximated as, $s_{A}=s_{2}+s_{4}+\cdots$, where $t_{2}$ vanishes at $s_{2}, t_{2}+t_{4}$ vanishes at $s_{2}+s_{4}$, and so on. On the right cut we can evaluate exactly $\operatorname{Im} 1 / t=-\sigma=-\operatorname{Im} t_{4} / t_{2}^{2}$, as can be read from Eqs. (1) and (3). Since the left cut is weighted at low energies we can use one-loop ChPT to approximate $L C(1 / t) \simeq L C\left(-t_{4} / t_{2}^{2}\right)$. The pole contribution $P C(1 / t)$ can be safely calculated with ChPT since it involves derivatives of $t$ evaluated at $s_{A}$, which is a low-energy point where ChPT is perfectly justified. Altogether, we arrive to a modified one-loop IAM (mIAM) formula [17]:

$$
\begin{aligned}
t^{\mathrm{mIAM}} & =\frac{t_{2}^{2}}{t_{2}-t_{4}+A^{\mathrm{mIAM}},} \\
A^{\mathrm{mIAM}} & =t_{4}\left(s_{2}\right)-\frac{\left(s_{2}-s_{A}\right)\left(s-s_{2}\right)\left[t_{2}^{\prime}\left(s_{2}\right)-t_{4}^{\prime}\left(s_{2}\right)\right]}{s-s_{A}},
\end{aligned}
$$

where the prime denotes the first derivative with respect to $s$ and where we use for $s_{A}$ in the numerical calculations its NLO approximation $s_{2}+s_{4}$. The standard IAM formula is 
recovered for $A^{\mathrm{mIAM}}=0$, which is indeed the case for all partial waves except the scalar ones. In the original IAM derivation $[15,16] A^{\mathrm{mIAM}}$ was neglected since it formally yields a higher order contribution and is numerically very small except near the Adler zero. However, if $A^{\text {mIAM }}$ is neglected, the IAM Adler zero occurs at $s_{2}$, correctly only to LO, is a double zero instead of a simple one, and a spurious pole appears close to the Adler zero. All of these caveats disappear with the mIAM, and the differences between the IAM and the mIAM in the physical and resonance region are of the order of $1 \%$.

It is important to remark that ChPT has not been used at all for calculations of $t(s)$ for positive energies above threshold. Note that the use of ChPT is well justified to calculate $s_{A}$, and $P C(1 / t)$, since these are low-energy calculations. ChPT has also been used to calculate the left cut integral, which, despite extending to infinity, is heavily weighted at low energies, which once again justifies the use of ChPT. The left cut and the elastic approximation are the only approximations used to obtain the IAM, but no other model dependent assumptions have been made. In particular there are no spurious parameters included in the IAM derivation, but just the ChPT LECs, $m_{\pi}$ and $f_{\pi}$.

Remarkably, these simple equations (either the IAM or the mIAM) ensure elastic unitarity, match ChPT at low energies and, using LECs compatible with existing determinations, describe fairly well data up to somewhat less than $1 \mathrm{GeV}$, generating the $\rho, K^{*}, \sigma$, and $\kappa$ resonances as poles on the second Riemann sheet [16].

The extension to two loops is very similar and straightforward for the IAM [16,42] or the mIAM [21]:

$$
\begin{aligned}
t^{\mathrm{mIAM}}= & \frac{t_{2}^{2}}{t_{2}-t_{4}+t_{4}^{2} / t_{2}-t_{6}+A^{\mathrm{mIAM}}}, \\
A^{\mathrm{mIAM}}= & t_{4}\left(s_{2}\right)-\frac{2 t_{4}\left(s_{2}\right) t_{4}^{\prime}\left(s_{2}\right)}{t_{2}^{\prime}\left(s_{2}\right)}-\frac{t_{4}^{2}\left(s_{2}\right)}{t_{2}^{\prime}\left(s_{2}\right)\left(s-s_{2}\right)}+t_{6}\left(s_{2}\right) \\
& +\frac{\left(s-s_{2}\right)\left(s_{A}-s_{2}\right)}{s-s_{A}}\left(t_{2}^{\prime}\left(s_{2}\right)-t_{4}^{\prime}\left(s_{2}\right)-t_{6}^{\prime}\left(s_{2}\right)\right. \\
& \left.+\frac{t_{4}^{\prime}\left(s_{2}\right)^{2}+t_{4}^{\prime \prime}\left(s_{2}\right) t_{4}\left(s_{2}\right)}{t_{2}^{\prime}\left(s_{2}\right)}\right)
\end{aligned}
$$

Let us now remark that both in the one and two-loop derivations above, we have assumed that $t_{2}$ is not identically zero. However, this is only the case for scalar and vector partial waves. Unfortunately, as seen in Eq. (3), when $t_{2}(s) \equiv 0$ the first imaginary part appears at $O\left(p^{8}\right)$, namely, at three loops. Therefore, we cannot recast the dispersion relation in terms of the full ChPT expansion unless we make use of $t_{8}(s)$, a calculation that does not exist. In [43], and using only the $t_{8}$ term of the form $c s^{4}$, it was shown that the $f_{2}(1275)$ shape could be fairly well fitted with the IAM and a $c$ value of the correct order of magnitude expected from dimensional grounds. This was justified because the $f_{2}$ resonance appears at high $s \gg m_{\pi}^{2}$ and the other $O\left(p^{8}\right)$ terms, containing pion mass powers, could be neglected. However, in this work we want to make $m_{\pi}$ much larger than its physical value and we need the $m_{\pi}$ dependence. It is, therefore, not so well justified to neglect all the $t_{8}$ terms except $c s^{4}$. For that reason, we are limited to use the IAM for scalar and vector partial waves.

Hence, using the IAM or the mIAM, we can study how the generated $\rho$ and $\sigma$ poles evolve by changing $m_{\pi}$ in the one-loop IAM amplitudes [18] or two-loop amplitudes [21], and describe the dependence of their masses, widths, and couplings on $m_{\pi}$. In [18] the mIAM was used for the $\rho$ and $\sigma$ chiral extrapolation, because, for the scalar and at high $m_{\pi}$, one resonance pole gets near the IAM spurious pole, a problem that is nicely solved with the mIAM. Nevertheless, in the physical region and near the other generated poles, the differences between IAM and mIAM approaches are almost negligible, even for high pion masses.

Of course, the poles are not the only object of study on the lattice. Actually, lattice results are already available for phase shifts in $I=2$ channels, where no pole exists. Moreover, these channels were not studied in [18,21]. It is also very likely that lattice results on phase shifts for other channels will be available soon. For these reasons, we will now let $m_{\pi}$ vary within our unitarized ChPT expressions, with the aim of extending the phase-shift predictions based on ChPT, up to higher masses and momenta.

\section{RESULTS WITH THE IAM AND CHPT}

Let us first recall, as already explained in some of the very first works on the IAM [16], and repeated in many other instances $[21,44,45]$, that when the central values of the standard LECs are used, the IAM only improves ChPT up to a couple of hundred $\mathrm{MeV}$ higher and resonances are only reproduced qualitatively. For a semiquantitative description of resonances, which is what we will do next, one has to fit the data and the resulting LECs are slightly modified from those obtained from pure ChPT. Since the IAM contains contributions that count as higher order in ChPT (in particular, the numerically relevant $s$-channel logarithms), one would very naively expect the LECs from the one-loop IAM to lie somewhere in between the one and two-loop values from ChPT. This is actually observed, since the $O\left(p^{4}\right)$ IAM LECs in Table III lie somewhere between the one and two-loop analysis of pure ChPT listed in Table II, although closer to the ChPT $O\left(p^{4}\right)$ analysis in the two first rows of that table. In contrast, the $O\left(p^{4}\right)$ values of the LECs for the two-loop IAM in Table IV are closer to the two-loop analyses like that in Table I or those in the third and fourth row of Table II. Let us emphasize that the variation between the $O\left(p^{4}\right)$ LECs values between the one and two-loop analyses already occurs in pure ChPT - particularly for $l_{2}^{r}$. The IAM simply follows a similar pattern. 
TABLE III. LECs used in this work for the one-loop IAM, obtained from a fit to the dispersive data analysis of [34]. Both $l_{3}$ and $l_{4}$ are fixed to the standard values given in Table I. The scale is set to $\mu=770 \mathrm{MeV}$.

\begin{tabular}{cc}
\hline \hline$O\left(p^{4}\right)$ & LECs $\left(\times 10^{-3}\right)$ \\
\hline$l_{1}^{r}(\mu)$ & $-3.9 \pm 0.2$ \\
$l_{2}^{r}(\mu)$ & $4.3 \pm 0.4$ \\
$l_{3}^{r}(\mu)$ & $0.18 \pm 1.11$ \\
$l_{4}^{r}(\mu)$ & $6.17 \pm 1.39$ \\
\hline \hline
\end{tabular}

Before changing the pion mass, let us note that for the IAM, we are assuming the elastic approximation and therefore, when increasing $m_{\pi}$, we should allow for some $\pi \pi$ elastic regime, which is guaranteed if $m_{\pi}<500 \mathrm{MeV}$, although it has been found that relatively stable unitarized results can be obtained for all waves only up to $m_{\pi} \simeq$ 300-350 MeV [21]. Of course, some waves are more stable than others. In particular, the elastic IAM approximation is quite good up to larger energies for the $(I, J)=$ $(2,0)$ (roughly up to $\sqrt{s} \simeq 1200-1300$, see [44]), since it has no resonances and does not couple to $\bar{K} K$. We will actually check that for this channel we can stretch the applicability range and still get fairly good agreement with recent lattice results for relatively large pion masses.

\section{A. One-loop IAM}

In Fig. 7 we show the IAM results to one-loop in ChPT, using the LECs in Table III, obtained by an updated fit to

TABLE IV. Low-energy constants obtained from fits [21] to experimental data on elastic $\pi \pi$ scattering and lattice results on $f_{\pi}, M_{\rho}$ and the isospin 2 scattering length as well as a $1 / N_{c}$ leading behavior of a pure $\bar{q} q$ state for the $\rho(770)$. Many of these sets are not quite compatible with each other and suffer large systematic uncertainties. These two fits correspond to different ways of weighting the existing experimental and lattice data sets, which are detailed in [21]. The values correspond to the scale $\mu=770 \mathrm{MeV}$.

\begin{tabular}{lrr}
\hline \hline & Set A & Set D \\
\hline$O\left(p^{4}\right)\left(\times 10^{-3}\right)$ & & \\
$l_{1}^{r}(\mu)$ & -5.0 & -4.0 \\
$l_{2}^{r}(\mu)$ & 1.7 & 1.2 \\
$l_{3}^{r}(\mu)$ & 0.8 & 0.8 \\
$l_{4}^{r}(\mu)$ & 6.5 & 6.5 \\
$O\left(p^{6}\right)\left(\times 10^{-4}\right)$ & & \\
$r_{1}^{r}(\mu)$ & -0.6 & -0.6 \\
$r_{2}^{r}(\mu)$ & 1.3 & 1.5 \\
$r_{3}^{r}(\mu)$ & -1.7 & -3.3 \\
$r_{4}^{r}(\mu)$ & 2.0 & 0.9 \\
$r_{5}^{r}(\mu)$ & 2.0 & 1.7 \\
$r_{6}^{r}(\mu)$ & -0.6 & -0.7 \\
$r_{f}^{r}(\mu)$ & -1.4 & -1.8 \\
\hline \hline
\end{tabular}
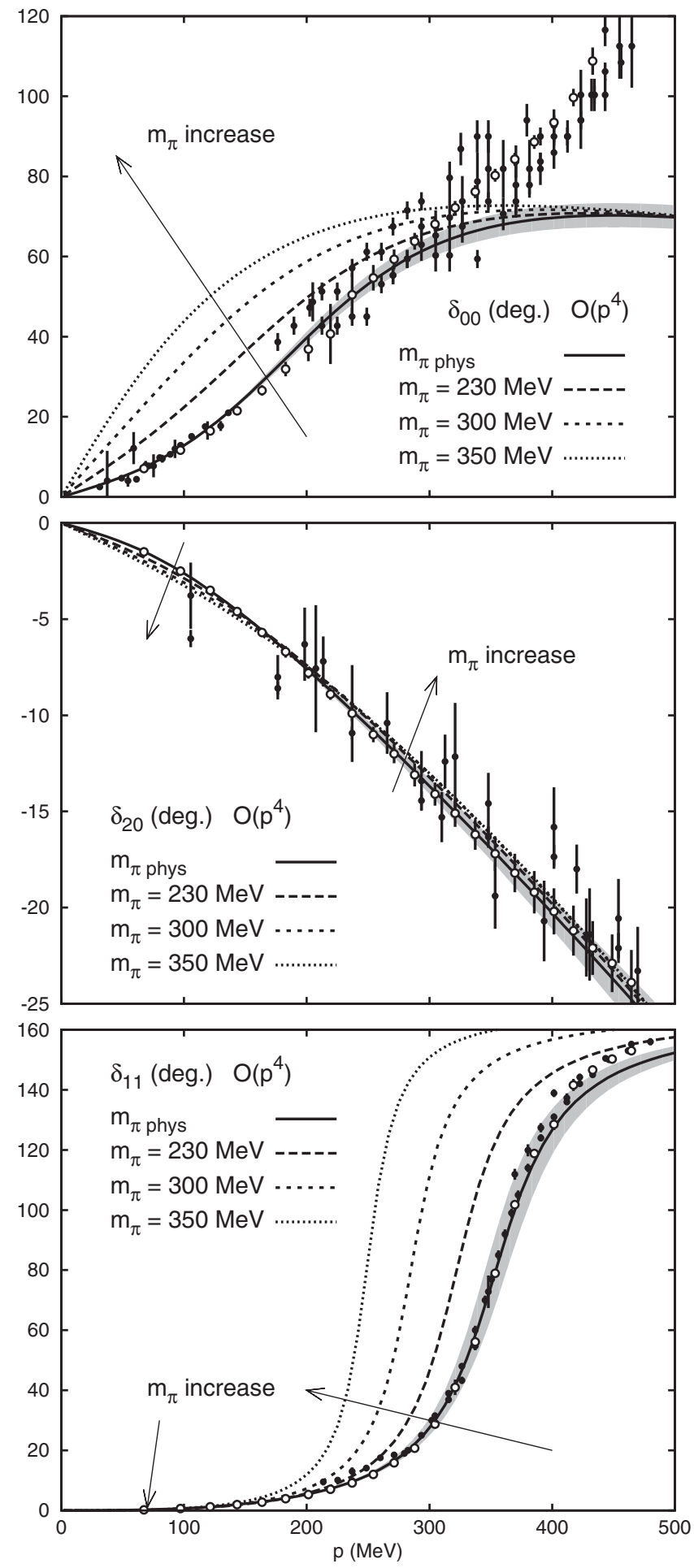

FIG. 7. $S$ and $P$ wave $\pi \pi$ phase shifts from unitarized ChPT up to one loop. Different lines stand for different pion masses: continuous, long dashed, short dashed and dotted for $M_{\pi}=$ 139.57, 230, 300 and $350 \mathrm{MeV}$, respectively. Since the lines are too close to each other, we only show error bands for the physical mass. Experimental data come from [33] (black circles) and the precise model independent dispersive data analysis from [34] (white circles). The arrows show the direction of increasing $m_{\pi}$. See Fig. 8 for a blow up of the low momentum region of the $I=1, J=1$ phase shift. 
the output from the recent and precise dispersive data analysis in [34], and fixing $l_{3}$ and $l_{4}$ to the updated values in Table I. The uncertainties are mostly systematic, arising from different choices of the maximum energy up to where we make the fit of the $(0,0)$ channel, which we have chosen between 500 and $800 \mathrm{MeV}$; the other channels are fitted up to $1 \mathrm{GeV}$. Note that the resulting LECs are consistent within 1 standard deviation with the results we used in [18], that we list in the last row of Table II. We first note that the experimental data is fairly well described up to the region where inelastic effects (or resonances like the $\left.f_{0}(980)\right)$ become relevant. This includes the $\rho(770)$ resonance shape, but also the wide shape of the $f_{0}(600)$. The gray bands in the figures cover the uncertainties in our results obtained from a Monte Carlo Gaussian sampling of the $l_{i}$ statistical error bars also listed in the table. As usual, and to avoid confusion due to many overlapping gray bands, we only show the uncertainty for the physical pion mass. Details on uncertainties for higher masses can be found in the appendix.

The general features for the scalar-isoscalar channel are very similar to the one-loop nonunitarized results. Namely, the phase shift conserve its positive sign and increases in absolute value as $m_{\pi}$ grows.

However, the $I=2$ channel behavior is rather different. First, the $m_{\pi}$ dependence is even milder than for the nonunitarized case. In the very low momentum region, roughly below $p=200 \mathrm{MeV}$, the phase increases in absolute value as it happened with standard one-loop ChPT. However, for larger momentum, the $m_{\pi}$ dependence is the opposite, and the phase starts decreasing its absolute value. As we will see later on, this is the behavior found on recent lattice results, which cannot be reproduced by a crude extrapolation of one-loop ChPT to larger momentum.

Something similar occurs in the vector channel, although enhanced by the presence of the $\rho(770)$ resonance that ChPT failed to reproduce. Now we see that the phase increases as the two-pion threshold grows and gets closer to the resonance. This is the intuitive behavior one would expect when getting close to the resonance. However, one should observe that it is not incompatible with the phase decrease observed in standard ChPT at low energies. To see this, in Fig. 8, we show a blow up of the very low-energy region of the vector channel, where we can see that the IAM behaves similarly to ChPT, namely, the phase decreases as $m_{\pi}$ grows. As explained before, this only happens in the very low momentum regime, since, as seen in the figure, for higher momentum the phase shift increases again since the IAM is able to reconstruct the $\rho(770)$ resonance, which is closer and closer to threshold as $m_{\pi}$ grows.

In the next subsection we will see that these general features and improvements with respect to nonunitarized $\mathrm{ChPT}$ are even more dramatic when considering the twoloop calculation.

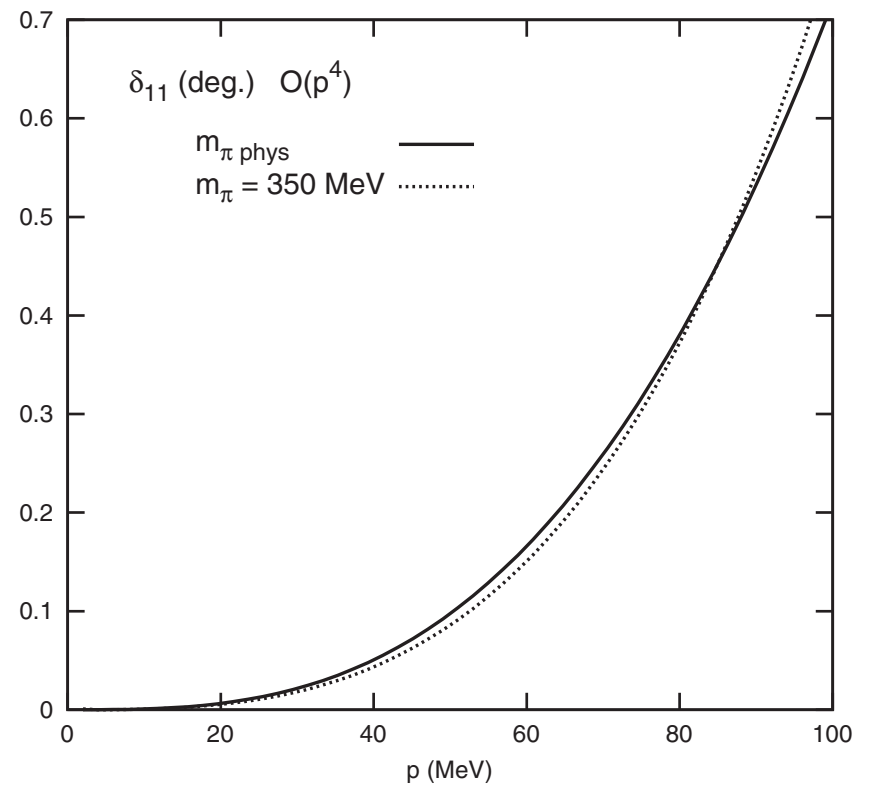

FIG. 8. $\pi \pi I=1, J=1$ phase shift from unitarized ChPT up to one loop. The continuous line stands for $M_{\pi}=139.57 \mathrm{MeV}$ and the dotted line for $M_{\pi}=350 \mathrm{MeV}$. Similarly to the ChPT case, in the low momentum region the phase shift decreases as $m_{\pi}$ grows. However at higher momentum it increases with the pion mass, due to the presence of the $\rho(770)$ resonance.

\section{B. Two-loop IAM}

In Figs. 9 and 10 we show the results of the two-loop IAM for the two best fits in [21], "A" and "D", whose corresponding sets of LECs we provide in Table IV. These fits have been obtained from an IAM fit to experimental data but also to lattice results on $f_{\pi}, M_{\rho}$ and the isospin 2 scattering length. Note that by fitting only the experimental data one determines better the LECs that govern the $s$ dependence, but not so well those governing the $m_{\pi}$ dependence. That is the reason why some existing lattice results on $f_{\pi}, M_{\rho}$ and the $I=2$ scalar scattering length were also included in the fits of [21]. Unfortunately, the experimental data in the resonance region are frequently in conflict with one another, and to a lesser extent, something similar happens for the lattice results mentioned above. Fits A and D correspond to different ways of weighting the conflicting experimental and lattice results, including some educated estimates for systematic uncertainties. The details can be found in [21]. These fits give rather stable results for all observables in the elastic region, up to $m_{\pi}=$ $300-350 \mathrm{MeV}$, and somewhat beyond for some particular waves, like $(I, J)=(2,0)$.

Note that the qualitative behavior of all waves is similar in Figs. 9 and 10. The difference between fit A and D is purely quantitative: in fit $\mathrm{A}$ the $m_{\pi}$ dependence is just stronger than in fit D.

Remarkably, almost all the features described for the one-loop unitarized case remain in the two-loop unitarized fits. Quantitatively, there are small differences, since the 
J. NEBREDA, J. R. PELÁEZ, AND G. RÍOS
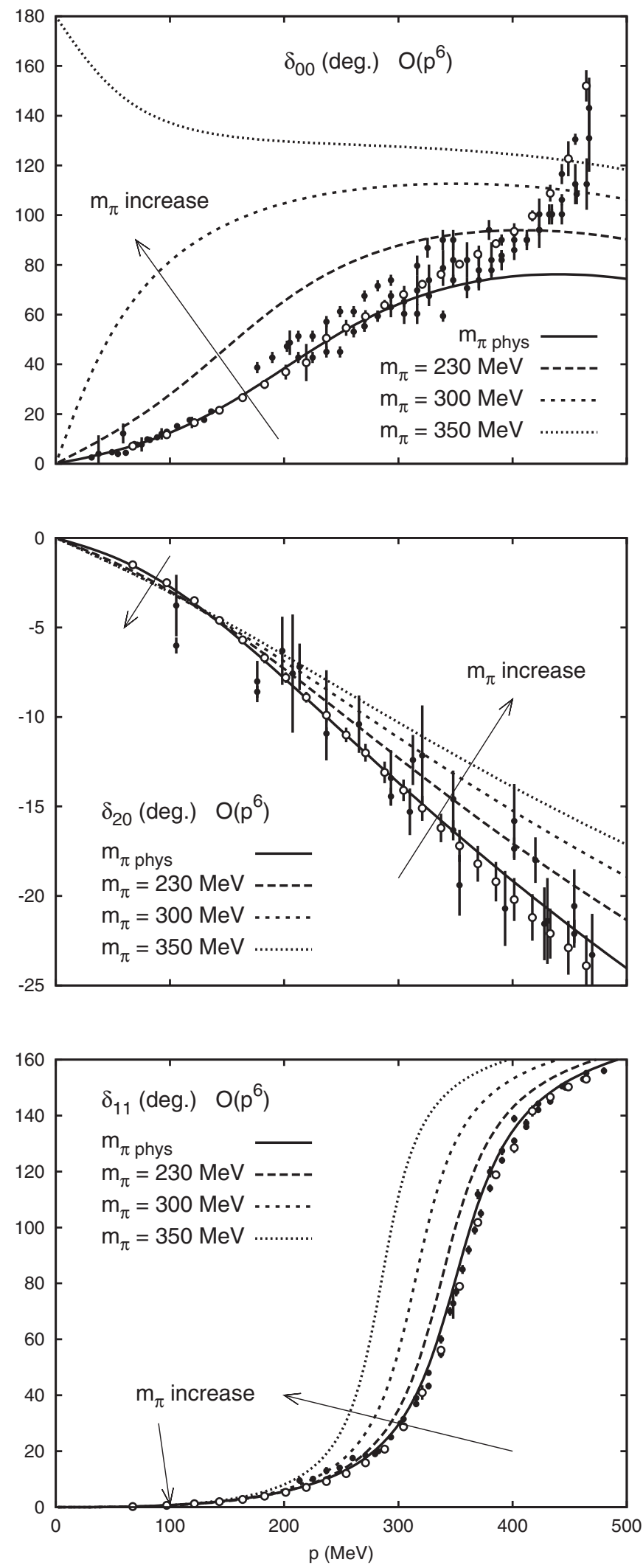

FIG. 9. $S$ and $P$ wave $\pi \pi$ phase shifts from the two-loop IAM "fit A" in [21]. The conventions are as in Fig. 7. The arrows show the direction of increasing $m_{\pi}$. The difference between these curves and those in Fig. 10 are an indication of the order of magnitude of our uncertainties.
PHYSICAL REVIEW D 83, 094011 (2011)
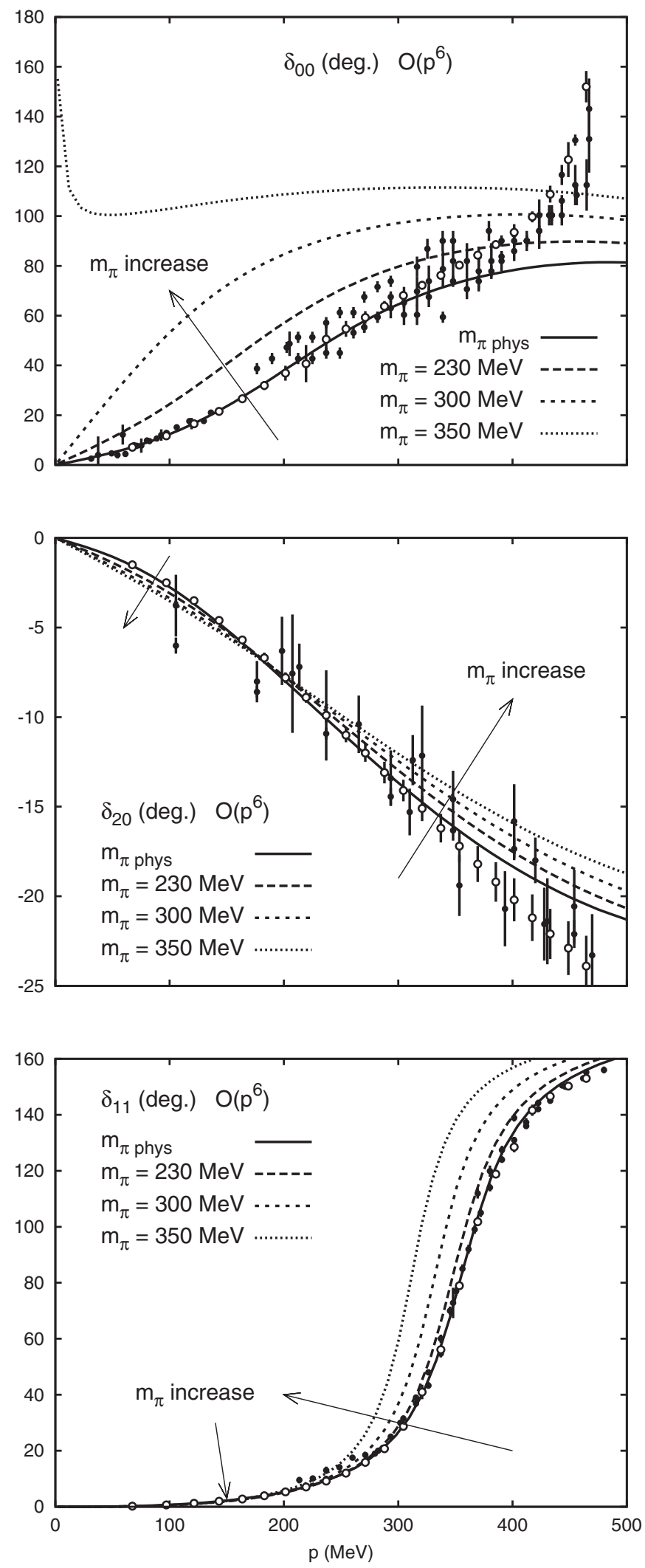

FIG. 10. $S$ and $P$ wave $\pi \pi$ phase shifts from the two-loop IAM "fit D" in [21]. The conventions are as in Fig. 7. The arrows show the direction of increasing $m_{\pi}$. The difference between these curves and those in Fig. 9 are an indication of the order of magnitude of our uncertainties. 
$m_{\pi}$ dependence at two loops seems somewhat stronger in the scalar waves, and somewhat weaker in the vector channel. This somewhat stronger $m_{\pi}$ dependence produces the only significant, and relevant, difference with the oneloop IAM. Both the one and two-loop IAM generate the $f_{0}(600)$ or $\sigma$ resonance as a pole deep in the complex plane, which mass grows much slower than the two-pion threshold, so that the "'bump" that this wide resonance produces in the $(0,0)$ phase is bigger and gets closer to threshold. Actually, as shown in [18] the two conjugated poles of the $f_{0}(600)$ move in the second, unphysical, Riemann sheet, until they reach the real axis below threshold, where the two poles are no longer conjugated. As $m_{\pi}$ keeps growing one of them jumps into the first Riemann sheet below threshold becoming a bound state. By Levinson's theorem [46], this implies that the phase at threshold increases by $\pi$. For the IAM to one-loop this jump occurs for $m_{\pi}$ larger than $350 \mathrm{MeV}$, but since the $m_{\pi}$ dependence is stronger for the IAM at two-loops, this jump can already be seen in Figs. 9 and 10 for the $m_{\pi}=$ $350 \mathrm{MeV}$ curve, which behavior thus reflects the existence of a bound state. Let us emphasize that the same behavior would be observed to one loop—although for higher $m_{\pi}$ but it will never be seen in standard ChPT, which cannot generate a pole.

However, when comparing with the nonunitarized twoloop results in Fig. 3, we see that unitarization not only improves the vector channel by describing the $\rho(770)$ resonance, but also the $I=2$ channel is nicely described up to much higher momentum, even though this channel is nonresonant. We will profit from this lack of complicated resonant structures in the $I=2$ scalar wave, and also from the fact that this channel does not couple to $\bar{K} K$, to extrapolate to higher pion masses where we will see that the unitarized results are in much better agreement than standard ChPT with some recent lattice results.

\section{COMPARISON WITH LATTICE RESULTS FOR$$
I=2 \text { AND } m_{\pi}>350 \mathrm{MeV}
$$

In Fig. 11 we show the results from the one and two-loop IAM with very recent results on the lattice $[12,13]$ for the $I=2$ scalar channel. Note that the data below $p=$ $200 \mathrm{MeV}$ is still fairly well described by the IAM, as it happened with ChPT, but that the IAM is not bending down and getting away from higher momentum data as it happened with standard ChPT results. Actually, the IAM results follow qualitatively the shape of the lattice data. Moreover, the $m_{\pi}$ dependence is much milder than for plain ChPT, in better agreement with the findings on the lattice. Let us remark that we do not aim at precision here because pion masses of $400 \mathrm{MeV}$ are probably close to the IAM applicability bound. Our approach should become more reliable below 300-350 MeV, where we expect lattice
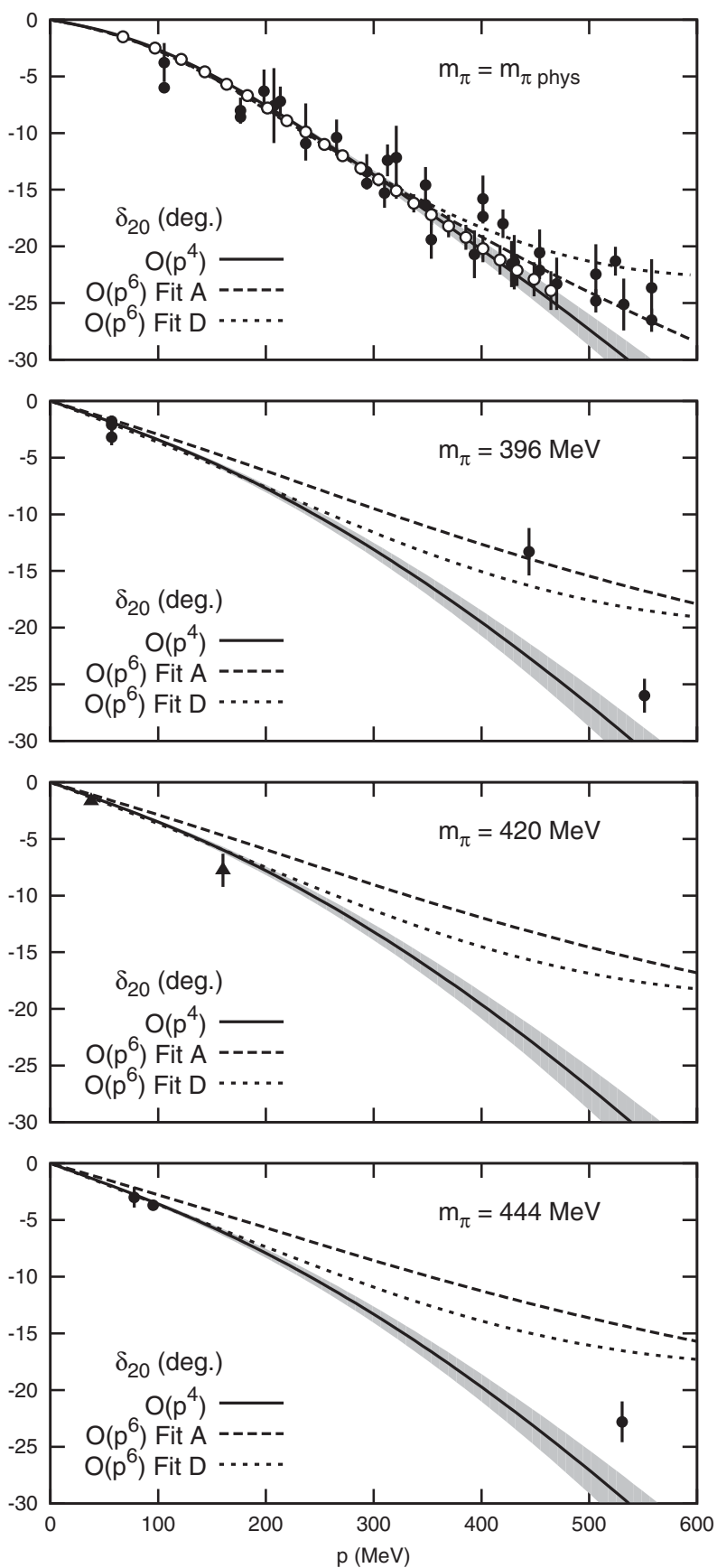

FIG. 11. One and two-loop IAM phase shifts for the $I=2$, $J=0$ channel. Top panel: results compared to data from [33] (black circles) and [34] (white circles). Rest of panels: results compared to lattice results coming from [12] (circles) and [13] (triangles). Note that for the two-loop case we provide results for the two best fits, A and D, obtained in [21].

results to appear soon. Still, the remarkable improvement with respect to the standard ChPT results is pretty clear.

As previously commented, the IAM cannot be directly applied to the D waves, since their tree level contribution vanishes. Further modifications of the IAM would be needed, which are beyond the scope of this work. 


\section{SUMMARY AND DISCUSSION}

In this work, we have studied the pion mass dependence of $\pi \pi$ elastic scattering phase shifts.

On the one hand, we have presented results for one and two-loop standard Chiral Perturbation Theory using a set of LECs obtained from a dispersive analysis in the literature. We have seen that this first approach is, of course, limited to low momentum, say below $300 \mathrm{MeV}$, depending on the channel, and pion masses up to $400-450 \mathrm{MeV}$. For the scalar and vector waves, we have found a rather stable behavior between the one and two-loop calculations within that momentum range. We have seen that at this very low momentum, the absolute value of scalar phase shifts increases as the pion mass grows, so that these channels enhance their attractive or repulsive nature. We have found that up to momenta less than $200 \mathrm{MeV}$, the ChPT results are in fair agreement with lattice data for the scalar $I=2$ channel.

We have found that, surprisingly, the vector phase shift at very low momentum decreases as $m_{\pi}$ grows within the applicability region. This may seem counterintuitive, since from lattice and other effective theory techniques, as $m_{\pi}$ grows one expects the two-pion threshold to approach fast the $\rho(770)$ mass. We have, nevertheless, shown with a very simple and intuitive model why very basic requirements about chiral symmetry impose such a decrease on the phase for low momentum and not too large $m_{\pi}$.

We have also shown results within standard ChPT for the angular momentum 2 phase shifts. These are much less stable when comparing one and two-loop results. Particularly for the $(I, J)=(2,2)$ channel, the one and two-loop results show an opposite behavior, and the twoloop calculation is also at odds with the $m_{\pi}$ dependence found on the lattice. Of course, one has to keep in mind that for $D$ waves, the one and two-loop calculations correspond to leading and next to leading order calculations, contrary to scalar and vector channels, where they correspond to next to leading and next to next to leading calculations. It is very likely that higher order calculations, or better determinations of LECs, which are highly correlated, may improve this situation for $D$ waves.

Finally, we have used ChPT inside a dispersion relation to extend the analysis of scalar and vector waves to higher momentum by means of the so called inverse amplitude method. This unitarization technique describes remarkably well the data up to energies of the order of 1 or $1.2 \mathrm{GeV}$, depending on the channel and has been shown to describe well the $m_{\pi}$ dependence of several observables like $M_{\rho}, f_{\pi}$ or the $I=2$ scalar scattering length.

The description provided by this method is, of course, compatible with that of standard ChPT at very low momentum. However, at higher momentum it reconstructs the behavior of the $\rho(770)$ resonance, which, for a given choice of low momentum, translates into a decreasing phase for smaller $m_{\pi}$ but a growing phase for larger $m_{\pi}$ until the $\rho(770)$ mass coincides with that particular momentum choice. In addition, we have shown that the unitarized $I=2$ scalar phase shift has the correct qualitative behavior for momentum beyond $200 \mathrm{MeV}$. Despite being close to the applicability bounds of the approach, we have actually shown that the IAM beyond $p=150-200 \mathrm{MeV}$ improves dramatically the description of lattice results with respect to ChPT and explains their very mild $m_{\pi}$ dependence.

Intuitively, the phase-shift evolution of the $S 0$ and $P$ channels is dominated by the presence of the $f_{0}(600)$ and $\rho(770)$ resonances and their pion mass dependence, studied in [18,20-22]. Since the masses of both resonances seem to grow slower than the pion mass, they come closer and closer to threshold so that, naively, one would expect the interaction to grow stronger and the phase to raise once the resonance is sufficiently close to the momentum where the phase is measured. Actually, this is what is found for the $S 0$ channel, whose phase raises noticeably as $m_{\pi}$ grows. At the limit of the range of applicability of the two-loop IAM, the $f_{0}(600)$ even becomes a bound state and by Levinson's theorem we see the phase to increase by $\pi$ at threshold. However, the naive expectations may not be met if the resonance is still not close enough to threshold. In such case, the phase may seem to decrease at first due to the finite size of the resonance, the effect of which has been illustrated in a simple model of the $\rho(770)$. Only when the $\rho(770)$ is sufficiently close to threshold, the naively expected behavior is observed. Concerning the $S 2$ wave, we have found a very mild $m_{\pi}$ dependence for the phase shift, when expressed in terms of the momentum, in good agreement with recent lattice calculations. This can be understood from the absence of resonant structures in this channel. Of course, ChPT can only reproduce the lowenergy tails of the resonances, which we have generated by means of ChPT unitarized with the IAM. For the D waves, the IAM cannot be applied to this order, and we have to rely on ChPT only. However, the behavior observed can also be understood from the presence of the $f_{2}(1270)$ resonance in the $D 0$ channel, and a similar behavior to the $\rho(770)$ in its own channel. For the $D 2$ channel, the ChPT results are not sufficiently precise to make any conclusive statement.

Apart from understanding the dependence of these observables on QCD parameters on the pion mass, we consider that this work is of interest as a guideline for future studies of lattice QCD.

\section{ACKNOWLEDGMENTS}

We thank J. Dudek for lattice results and detailed explanations. Our work was partially supported by the Spanish Ministerio de Educación y Ciencia research Contract Nos.: FPA2007-29115-E, FPA2008-00592 and FIS2006-03438, and U. Complutense/Banco Santander 


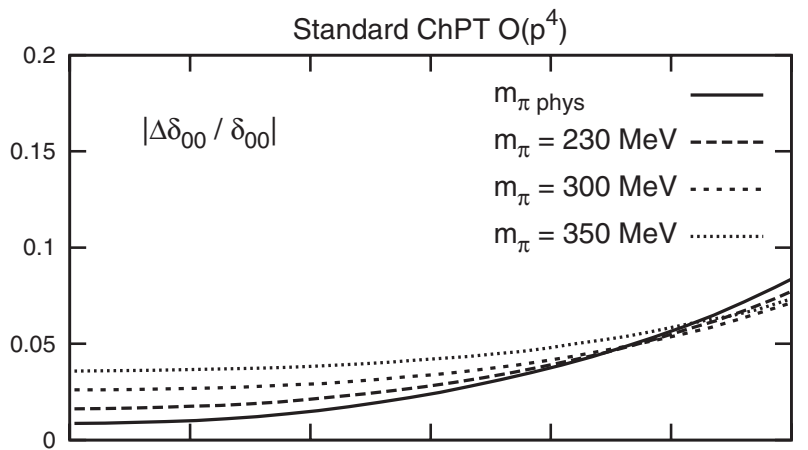

Standard ChPT O $\left(p^{6}\right)$
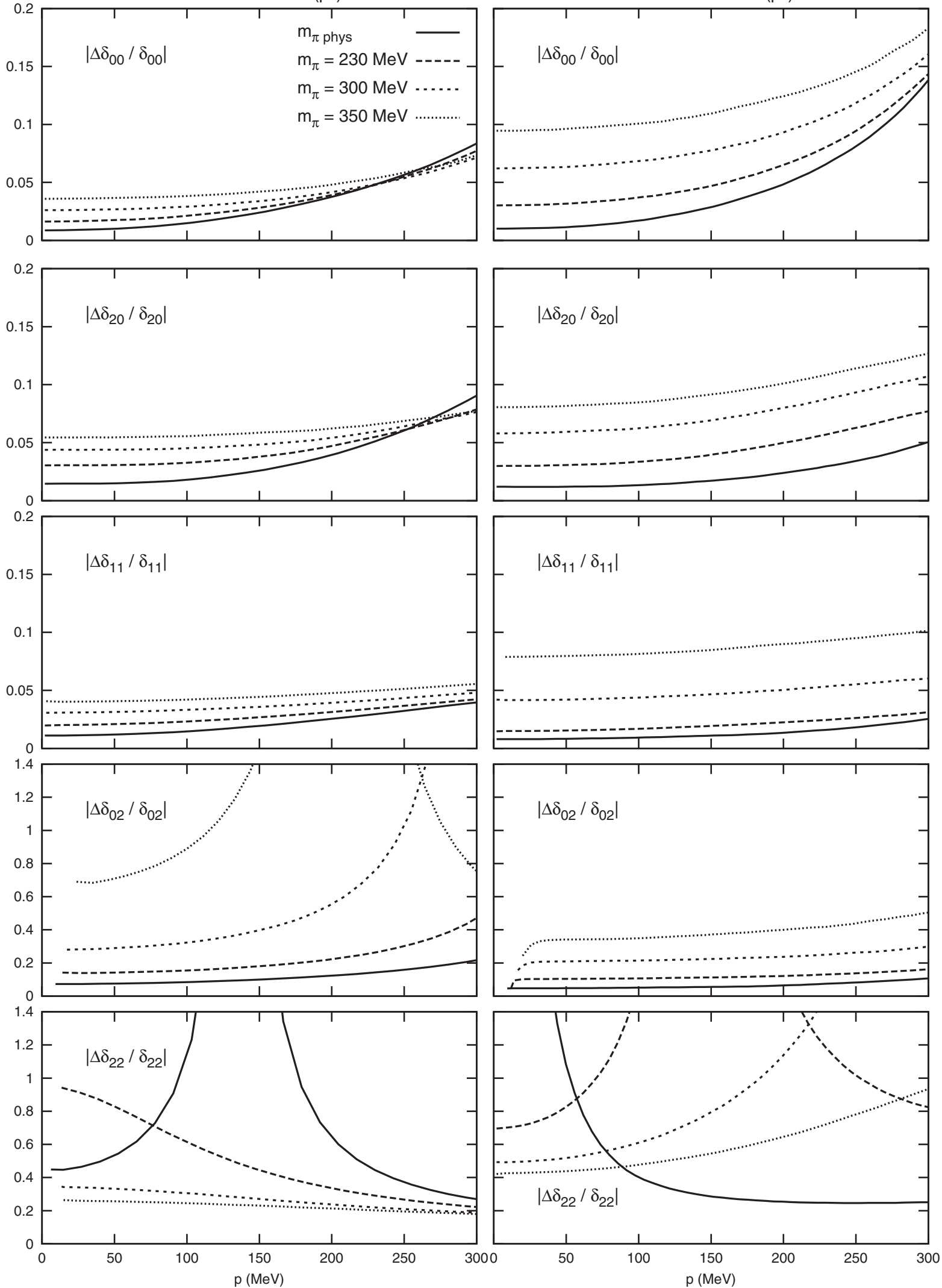

FIG. 12. $\pi \pi$ phase shift errors normalized to the value of the phase shifts in standard ChPT to one loop (left column) and to two loops (right column). Different lines stand for different pion masses: continuous, long dashed, short dashed and dotted for $m_{\pi}=139.57,230,300$ and $350 \mathrm{MeV}$, respectively. 

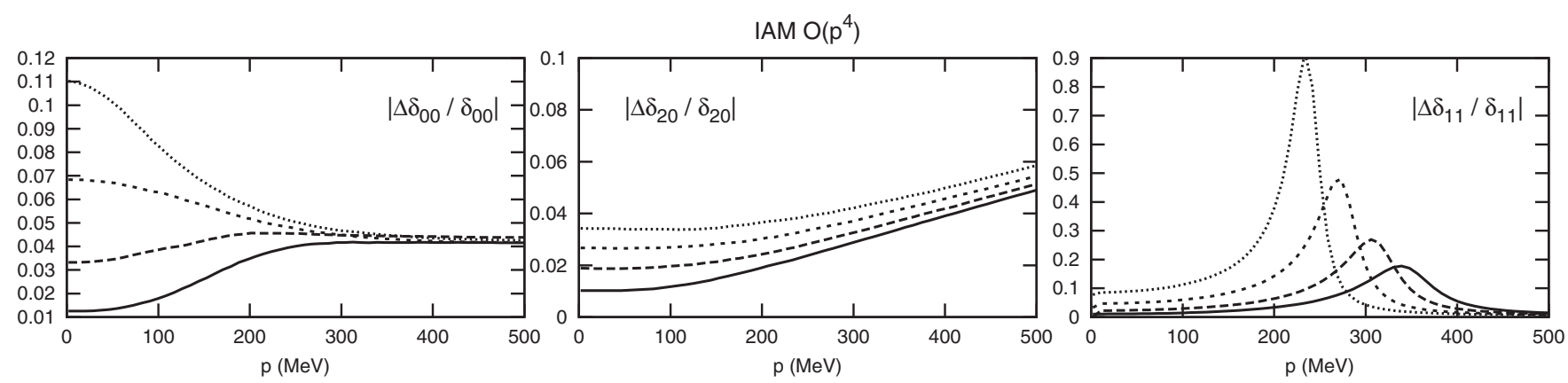

FIG. 13. $\pi \pi$ phase shift errors normalized to the value of the phase shifts in unitarized ChPT to one loop. Different lines stand for different pion masses: continuous, long dashed, short dashed and dotted for $m_{\pi}=139.57,230$, 300 and $350 \mathrm{MeV}$, respectively.

Grant Nos. PR34/07-15875-BSCH and UCM-BSCH GR58/08 910309. We acknowledge the support of the European Community-Research Infrastructure Integrating Activity Study of Strongly Interacting Matter (acronym HadronPhysics2, Grant Agreement No. 227431) under the Seventh Framework Programme of the European Union.

\section{APPENDIX: PHASE-SHIFT UNCERTAINTIES FOR DIFFERENT $\boldsymbol{m}_{\pi}$}

In Fig. 12, we plot the relative uncertainties of the standard ChPT phase-shift calculation. As we have already seen, standard ChPT is limited to low momentum and thus we only show momentum up to $p=300 \mathrm{MeV}$. For the scalar and vector waves we see that in the low momentum region the errors grow with the pion mass. This is in agreement with the fact that the LECs that govern the mass dependence of the partial waves carry the biggest uncertainties. For $D$-waves, the relative uncertainty is much bigger than for lower angular momentum waves. (Note the difference in scales between the $D$ waves and the rest of the plots). This is due to the fact that for $D$-waves the tree level calculation vanishes and therefore the one and two-loop results are just leading and next to leading order. In the case of $\delta_{02}$ to one loop, the error seems to explode for the highest masses due to the phase shift changing from a positive to a negative value in the region of interest. The same occurs for $\delta_{22}$ to one loop for the physical value of the pion mass. Finally, the value of $\delta_{22}$ to two loops changes from negative to positive for the lightest masses of the pion.

In Fig. 13, we show the relative uncertainties for the IAM phase shifts. We find again that for scalar waves they grow bigger as the pion mass is increased. The same happens for the vector phase shift below the $\rho(770)$ peak. The highest uncertainty on $\delta_{11}$ occurs when the slope of the phase shift reaches its maximum value.
[1] M.H. Johnson and E. Teller, Phys. Rev. 98, 783 (1955).

[2] K. Nakamura et al. (Particle Data Group), J. Phys. G 37, 075021 (2010).

[3] G. Colangelo, J. Gasser, and H. Leutwyler, Phys. Rev. Lett. 86, 5008 (2001).

[4] C. W. Bernard et al., Phys. Rev. D 64, 054506 (2001).

[5] Ph. Boucaud et al. (ETM Collaboration), Phys. Lett. B 650, 304 (2007).

[6] C. Allton et al. (RBC and UKQCD Collaborations), Phys. Rev. D 76, 014504 (2007).

[7] C. R. Allton et al., Phys. Lett. B 628, 125 (2005).

[8] M. Gockeler et al. (QCDSF Collaboration), Proc. Sci., LAT2008 (2008) 136;J. Noaki et al., Proc. Sci., LAT2008 (2008) 107 [arXiv:0810.1360].

[9] S. R. Beane et al., Phys. Rev. D 77, 014505 (2008).

[10] X. Feng, K. Jansen, and D. B. Renner, Phys. Lett. B 684, 268 (2010).

[11] J. Bulava, K. J. Juge, C. J. Morningstar, M. J. Peardon, and C.H. Wong, Proc. Sci., LAT2009 (2009) 097
[arXiv:0911.2044]; C. Morningstar et al., in HADRON 2009: Proceedings of the XIII International Conference on Hadron Spectroscopy, edited by Volker Crede, Paul Eugenio, and A. Ostrovidov, AIP Conf. Proc. No. 1257 (AIP, New York, 2010).

[12] J. J. Dudek, R. G. Edwards, M. J. Peardon, D. G. Richards, and C.E. Thomas, arXiv:1011.6352.

[13] K. Sasaki and N. Ishizuka, Phys. Rev. D 78, 014511 (2008).

[14] J. Gasser and H. Leutwyler, Ann. Phys. (N.Y.) 158, 142 (1984).

[15] T. N. Truong, Phys. Rev. Lett. 61, 2526 (1988); 67, 2260 (1991); A. Dobado et al., Phys. Lett. B 235, 134 (1990).

[16] A. Dobado and J. R. Peláez, Phys. Rev. D 47, 4883 (1993); 56, 3057 (1997).

[17] A. Gomez Nicola, J. R. Peláez, and G. Ríos, Phys. Rev. D 77, 056006 (2008).

[18] C. Hanhart, J. R. Peláez, and G. Ríos, Phys. Rev. Lett. 100, 152001 (2008). 
[19] J.F. Donoghue, Phys. Rev. C 74, 024002 (2006); T. Damour and J.F. Donoghue, Phys. Rev. D 78, 014014 (2008).

[20] J. Nebreda and J. R. Peláez, Phys. Rev. D 81, 054035 (2010).

[21] J. R. Peláez and G. Ríos, Phys. Rev. D 82, 114002 (2010).

[22] P.C. Bruns and U.-G. Meißner, Eur. Phys. J. C 40, 97 (2005).

[23] S. Weinberg, Physica A (Amsterdam) 96, 327 (1979).

[24] G. Colangelo, J. Gasser, and H. Leutwyler, Nucl. Phys. B603, 125 (2001).

[25] G. Colangelo et al., arXiv:1011.4408.

[26] G. Colangelo and S. Durr, Eur. Phys. J. C 33, 543 (2004).

[27] J. Bijnens, G. Colangelo, G. Ecker, J. Gasser, and M. E. Sainio, Nucl. Phys.B508, 263 (1997); B517, 639(E) (1998).

[28] J. Bijnens, Prog. Part. Nucl. Phys. 58, 521 (2007).

[29] P. Buettiker, S. Descotes-Genon, and B. Moussallam, Eur. Phys. J. C 33, 409 (2004); S. Descotes-Genon and B. Moussallam, Eur. Phys. J. C 48, 553 (2006).

[30] G. Amoros, J. Bijnens, and P. Talavera, Nucl. Phys. B602, 87 (2001).

[31] L. Girlanda, M. Knecht, B. Moussallam, and J. Stern, Phys. Lett. B 409, 461 (1997).

[32] J. Gasser and U. G. Meissner, Phys. Lett. B 258, 219 (1991).

[33] G. Grayer et al., Nucl. Phys. B75, 189 (1974); L. Rosselet et al., Phys. Rev. D 15, 574 (1977); S. Pislak et al. (BNLE865 Collaboration), Phys. Rev. Lett. 87, 221801 (2001); J. R. Batley et al. (NA48/2 Collaboration), Eur. Phys. J. C 54, 411 (2008); W. Hoogland et al., Nucl. Phys. B126, 109 (1977); M. J. Losty et al., Nucl. Phys. B69, 185 (1974); N. B. Durusoy et al., Phys. Lett. B 45, 517 (1973); S. D. Protopopescu et al., Phys. Rev. D 7, 1279 (1973); P. Estabrooks and A.D. Martin, Nucl. Phys. B79, 301
(1974); B. Hyams et al., Nucl. Phys. B64, 134 (1973); D. H. Cohen et al., Phys. Rev. D 7, 661 (1973).

[34] R. Garcia-Martín, R. Kamiński, J. R. Peláez, J. R. de Elvira, and F. J. Ynduráin, Phys. Rev. D 83, 074004 (2011).

[35] J. Nebreda, G. Ríos, J. R. Peláez, and F. J. Ynduráin (work in progress).

[36] P. C. Bruns and U.G. Meissner, Eur. Phys. J. C 40, 97 (2005).

[37] G. Ecker, J. Gasser, H. Leutwyler, A. Pich, and E. de Rafael, Phys. Lett. B 223, 425 (1989).

[38] F. Von Hippel and C. Quigg, Phys. Rev. D 5, 624 (1972); H. Becker et al. (CERN-Munich Collaboration), Nucl. Phys. B150, 301 (1979); V. Chabaud et al. (CERNCracow-Munich Collaboration), Nucl. Phys. B223, 1 (1983); R. Kaminski, L. Lesniak, and K. Rybicki, Z. Phys. C 74, 79 (1997); S. Kopp et al. (CLEO Collaboration), Phys. Rev. D 63, 092001 (2001).

[39] J. Blatt and V.F. Weisskopf, Theoretical Nuclear Physics (Wiley, New York, 1952), p. 361.

[40] M.A.B. Beg and A. Zepeda, Phys. Rev. D 6, 2912 (1972).

[41] M. K. Volkov and V. N. Pervushin, Nuovo Cimento Soc. Ital. Fis. A 27, 277 (1975).

[42] J. Nieves, M. Pavon Valderrama, and E. Ruiz Arriola, Phys. Rev. D 65, 036002 (2002).

[43] A. Dobado and J. R. Pelaez, Phys. Rev. D 65, 077502 (2002).

[44] A. Gomez Nicola and J. R. Peláez, Phys. Rev. D 65, 054009 (2002); J.R. Peláez, Mod. Phys. Lett. A 19, 2879 (2004).

[45] J. R. Peláez, AIP Conf. Proc. 892, 72 (2007); H. Leutwyler, AIP Conf. Proc. 1030, 46 (2008).

[46] N. Levinson, K. Dan. Vidensk. Selsk. Mat. Fys. Medd. 25, 9 (1949); R. G. Newton, Scattering Theory of Waves and Particles (McGraw-Hilll, New York, 1966). 